\title{
COURBES ELLIPTIQUES AYANT BONNE RÉDUCTION EN DEHORS DE 3
}

\author{
GÉRARD LIGOZAT
}

\section{Introduction}

Le résultat principal de ce travail est le suivant: les courbes elliptiques définies sur $\boldsymbol{Q}$, et ayant bonne réduction en dehors de 3, vérifient la conjecture de Weil (cf. [2], Th. 2).

La démonstration de ce fait découle d'une construction explicite des formes paraboliques de poids 2 sur les groupes de congruence $\Gamma_{0}\left(3^{\nu}\right), \nu=$ $3,4,5$. La méthode utilisée pour faire cette construction, ainsi que la façon d'en déduire la validité de la conjecture de Weil, sont celles employées par Honda et Miyawaki dans leur article [2] relatif au cas analogue des courbes ayant bonne réduction en dehors de 2 .

Ces questions sont traitées dans les deux premiers paragraphes.

Dans un troisième paragraphe, on utilise les symboles modulaires pour donner une description de la jacobienne $J_{0}\left(3^{4}\right)$, associée au groupe de congruence $\Gamma_{0}\left(3^{4}\right)$, en termes de réseaux complexes. On montre dans le quatrième paragraphe comment on peut déduire des résultats obtenus: d'une part, certaines propriétés galoisiennes des points de 3-division des courbes elliptiques de conducteur 27; d'autre part, les propriétés galoisiennes des points de 3-division d'une certaine courbe elliptique considérée par Koike dans [3], §3. Ces propriétés sont démontrées par Koike par une autre méthode, et lui servent à déterminer une équation explicite de cette courbe. Notre démonstration montre qu'elles ont une origine "modulaire".

Je tiens à remercier ici le Professeur Koike qui a bien voulu s'intéresser aux résultats de la première partie de ce travail, et dont l'article cité plus haut a motivé la seconde partie.

Received October 16, 1978. 


\section{Construction de formes paraboliques}

Notons $\left\langle\Gamma_{0}\left(3^{\nu}\right), 2\right\rangle_{0}$ l'espace des formes paraboliques de poids 2 sur le groupe de congruence $\Gamma_{0}\left(3^{\nu}\right), \nu=3,4,5$, resp. Cet espace est de dimension $1,4,19$ resp. Le sous-espace engendré par les formes primitives est de dimension 1,2,12 resp.

Soit $\mathfrak{S}=\{z \in C|\operatorname{Im}(z)\rangle 0\}$ le demi-plan de Poincaré. On notera $\mathfrak{S}^{*}$ le demi-plan de Poincaré complété:

$$
\mathfrak{S}^{*}=\mathfrak{S} \cup \boldsymbol{Q} \cup\{\infty\} .
$$

On désigne par $X_{0}\left(3^{\nu}\right), \nu=3,4,5$, la courbe modulaire associée à $\Gamma_{0}\left(3^{\nu}\right)$. Cette courbe est lisse, projective, définie sur $\boldsymbol{Q}$, et de genre égal à la dimension de $\left\langle\Gamma_{0}\left(3^{\nu}\right), 2\right\rangle_{0}$. Enfin, on note $J_{0}\left(3^{\nu}\right)$ la jacobienne de $X_{0}\left(3^{\nu}\right)$.

Soit $\eta(z)$ la fonction êta de Dedekind, définie par

$$
\eta(z)=e^{\pi i z / 12} \prod_{n=1}^{\infty}\left(1-q^{n}\right),
$$

où $z \in \mathfrak{S}$, et où l'on pose $q=e^{2 \pi i z}$.

1.1. Formes $\operatorname{sur} \Gamma_{0}\left(3^{\nu}\right), \nu=3,4$

Proposition 1.1.1. a) La fonction:

$$
\begin{aligned}
F_{1}^{(3)}(z)= & \eta(3 z)^{2} \eta(9 z)^{2} \equiv q-2 q^{4}-q^{7}+5 q^{13}+4 q^{16}-7 q^{19} \\
& -5 q^{25}+2 q^{28}-4 q^{31}\left(\bmod q^{37}\right),
\end{aligned}
$$

est l'unique forme primitive normalisée de $\left\langle\Gamma_{0}\left(3^{3}\right), 2\right\rangle_{0}$.

b) Soient:

$$
\begin{aligned}
G_{1}^{(4)}(z)= & \eta(3 z) \eta(9 z)^{2} \eta(27 z) \equiv q^{2}-q^{5}-q^{8}-2 q^{11}+2 q^{14}+3 q^{17} \\
& -q^{20}+2 q^{23}-q^{26}+q^{29}-3 q^{32}-2 q^{35}\left(\bmod q^{38}\right),
\end{aligned}
$$

et

$$
\begin{aligned}
G_{2}^{(4)}(z)= & \eta(3 z)^{-1} \eta(9 z)^{6} \eta(27 z)^{-1} \equiv q+q^{4}+2 q^{7}-3 q^{10}-q^{13} \\
& -5 q^{16}+2 q^{19}-6 q^{22}-2 q^{25}+2 q^{28}+8 q^{31}+9 q^{34} \quad\left(\bmod q^{37}\right) .
\end{aligned}
$$

Les fonctions $G_{1}^{(4)}, G_{2}^{(4)}$ constituent une base de l'espace engendré dans $\left\langle\Gamma_{0}\left(3^{4}\right), 2\right\rangle_{0}$ par les formes primitives. Les deux formes primitives normalisées sont:

$$
F_{1}^{(4)}=G_{2}^{(4)}+\sqrt{3} \cdot G_{1}^{(4)}
$$

et 


$$
F_{2}^{(4)}=G_{2}^{(4)}-\sqrt{3} \cdot G_{1}^{(4)} .
$$

Démonstration. Le Théorème 1 de [2] montre que $F_{1}^{(3)}$ est un élément (non nul) de $\left\langle\Gamma_{0}\left(3^{3}\right), 2\right\rangle_{0}$ (cf. aussi [4], Prop. 3.1.1); on en déduit la partie (a); le même théorème entraîne que $G_{1}^{(4)}$ et $G_{2}^{(4)}$ sont des éléments non nuls de $\left\langle\Gamma_{0}\left(3^{4}\right), 2\right\rangle_{0}$.

Soit $S$ la matrice:

$$
S=\left(\begin{array}{cc}
1 & 0 \\
27 & 1
\end{array}\right)
$$

Toujours d'après [2], Th. A, on a, avec les conventions de loc. cit.:

$$
\begin{aligned}
\eta(3 \cdot S z) & =(27 z+1)^{1 / 2} \cdot e^{-3 \pi i / 4} \cdot \eta(3 z), \\
\eta(9 \cdot S z) & =(27 z+1)^{1 / 2} \cdot e^{-\pi i / 4} \cdot \eta(9 z), \\
\eta(27 \cdot S z) & =(27 z+1)^{1 / 2} \cdot e^{-\pi i / 12} \cdot \eta(27 z) .
\end{aligned}
$$

Il en résulte:

$$
\begin{aligned}
& \left.G_{1}^{(4)}\right|_{2} S=e^{-4 \pi i / 3} \cdot G_{1}^{(4)}, \\
& \left.G_{2}^{(4)}\right|_{2} S=e^{-2 \pi i / 3} \cdot G_{2}^{(4)} .
\end{aligned}
$$

Ceci entraîne (cf. [2], p. 368) que $G_{1}^{(4)}$ et $G_{2}^{(4)}$ sont orthogonaux à $\left\langle\Gamma_{0}(27), 2\right\rangle_{0}$ pour le produit scalaire de Petersson; ce sont donc des combinaisons linéaires de formes primitives de $\left\langle\Gamma_{0}\left(3^{4}\right), 2\right\rangle_{0}$.

Enfin, l'action de l'opérateur de Hecke $T(2)$ est la suivante:

$$
\begin{aligned}
& G_{1}^{(4)} \mid T(2)=G_{2}^{(4)}, \\
& G_{2}^{(4)} \mid T(2)=3 G_{1}^{(4)} .
\end{aligned}
$$

On obtient $F_{1}^{(4)}, F_{2}^{(4)}$ en diagonalisant.

1.2. Formes sur $\Gamma_{0}\left(3^{5}\right)$

Considérons maintenant l'espace $\left\langle\Gamma_{0}\left(3^{5}\right), 2\right\rangle_{0}$, et cherchons à déterminer les éléments de cet espace qui s'écrivent comme produits de fonctions êta. On obtient le résultat suivant:

Proposition 1.2.1. La fonction:

$$
\eta(z)^{n_{1}} \eta(3 z)^{n_{2}} \eta\left(3^{2} z\right)^{n_{3}} \eta\left(3^{3} z\right)^{n_{4}} \eta\left(3^{4} z\right)^{n_{5}} \eta\left(3^{5} z\right)^{n_{6}},
$$

où $n_{1}, \cdots, n_{6} \in Z$, est une forme parabolique de poids 2 sur $\Gamma_{0}\left(3^{5}\right)$ si et seulement si $\left(n_{1}, \cdots, n_{6}\right)$ prend l'une des seize valeurs données dans le tableau suivant. 


\begin{tabular}{|c|c|}
\hline$\left(n_{1}, n_{2}, n_{3}, n_{4}, n_{5}, n_{6}\right)$ & $\begin{array}{l}\text { notation de la fonction } \\
\text { correspondante }\end{array}$ \\
\hline$(0,-1,3,3,-1,0)$ & $G_{1}^{(5)}$ \\
\hline$(0,-1,5,-1,1,0)$ & $G_{2}^{(5)}$ \\
\hline$(0,-1,4,-1,2,0)$ & $G_{3}^{(5)}$ \\
\hline$(0,2,1,0,1,0)$ & $G_{4}^{(5)}$ \\
\hline$(0,2,0,0,2,0)$ & $G_{5}^{(5)}$ \\
\hline$(0,2,-1,4,-1,0)$ & $G_{6}^{(5)}$ \\
\hline$(0,1,-1,5,-1,0)$ & $G_{8}^{(5)}$ \\
\hline$(0,1,1,1,1,0)$ & $G_{9}^{(5)}$ \\
\hline$(0,1,0,1,2,0)$ & $G_{10}^{(5)}$ \\
\hline$(0,2,2,0,0,0)$ & $F_{1}^{(3)}$ \\
\hline$(0,0,2,2,0,0)$ & \\
\hline$(0,0,0,2,2,0)$ & \\
\hline$(0,1,2,1,0,0)$ & $G_{1}^{(4)}$ \\
\hline$(0,0,1,2,1,0)$ & \\
\hline$(0,-1,6,-1,0,0)$ & $G_{2}^{(4)}$ \\
\hline$(0,0,-1,6,-1,0)$ & \\
\hline
\end{tabular}

Les formes $G_{i}^{(5)}, 1 \leq i \leq 10, i \neq 7$, sont linéairement indépendantes, et appartiennent à l'espace engendré dans $\left\langle\Gamma_{0}\left(3^{5}\right), 2\right\rangle_{0}$ par les formes primitives.

Démonstration. Utilisons les résultats de [4], 3.2. D'après la Prop. 3.2.8 de loc. cit., l'ordre de la fonction définie par (1.2.1.1) en une pointe de niveau $3^{j-1}(1 \leq j \leq 6)$ est donné par la $j$-ième ligne de la matrice:

$$
\frac{1}{24} \Omega\left(\begin{array}{c}
n_{1} \\
\vdots \\
n_{6}
\end{array}\right)=\left(\begin{array}{c}
m_{1} \\
\vdots \\
m_{6}
\end{array}\right),
$$


où l'on désigne par $\Omega$ la matrice de terme:

$$
\Omega_{i, j}=3^{5+2 \inf (i, j)-\inf (i-1,6-i)-(i+j)} . \quad 1 \leq i, j \leq 6 .
$$

Le raisonnement fait dans la démonstration de la Prop. 3.1.1 de [4] montre que les conditions suivantes sont nécessaires et suffisantes pour que la fonction associée à $\left(n_{1}, \cdots, n_{6}\right)$ soit une forme parabolique de poids 2 sur $\Gamma_{0}\left(3^{5}\right)$ :

(i) $n_{1}+\cdots+n_{6}=4$;

(ii) $m_{1}, \cdots, m_{6} \in Z$;

(iii) $m_{1}, \cdots, m_{6}>0$;

(iv) $n_{1}+n_{3}+n_{5} \equiv 0 \quad(\bmod 2)$.

S'il en est ainsi, le diviseur de la forme différentielle associée à la forme parabolique est de degré 36 ; ceci s'exprime par la formule:

(v) $m_{1}+m_{6}+2\left(m_{2}+m_{5}\right)+6\left(m_{3}+m_{4}\right)=54$.

Pour déterminer les solutions du système (i, ii, iii, iv), il est commode d'utiliser (v). Plus précisément, posons:

$$
u=m_{1}+m_{6} ; \quad v=m_{2}+m_{5} ; \quad w=m_{3}+m_{4} .
$$

La condition (iii) entraîne:

(iii) $\quad u, v, w \geq 2$.

D'autre part, on tire de (1.2.1.2):

$$
\begin{aligned}
& 24 u=244 n_{1}+84 n_{2}+36 n_{3}+36 n_{4}+84 n_{5}+244 n_{6}, \\
& 24 v=28 n_{1}+84 n_{2}+36 n_{3}+36 n_{4}+84 n_{5}+28 n_{6}, \\
& 24 w=4 n_{1}+12 n_{2}+36 n_{3}+36 n_{4}+12 n_{5}+4 n_{6},
\end{aligned}
$$

d'où:

(vi) $\left\{\begin{array}{l}n_{1}+n_{6}=\frac{u-v}{9} \\ n_{3}+n_{4}=\frac{-v+7 w}{9} .\end{array}\right.$

Dans un premier temps, on détermine les triplets $(u, v, w)$ vérifiant simultanément:

(v) $u+2 v+6 w=54$; 
(iii) $\quad u, v, w \geq 2$;

(vi) $\quad u \equiv v(\bmod 9) ; \quad v \equiv 7 w(\bmod 9)$.

On trouve ainsi les neuf solutions suivantes:

$(2,2,8) ;(4,4,7) ;(6,6,6) ;(8,8,5) ;(10,10,4) ;(12,12,3) ;(14,14,2)$;

$(30,3,3) ;(32,5,2)$.

Pour chacune de ces neuf valeurs, on est ramené à résoudre un système ne faisant intervenir que la moitié des inconnues. Montrons comment on traite le cas $u=2, v=2, w=8$.

On a; $\quad m_{1}+m_{6}=2 ; m_{2}+m_{5}=2 ; m_{3}+m_{4}=8$,

soit $(*): \quad n_{1}+n_{6}=0 ; n_{2}+n_{5}=-2 ; n_{3}+n_{4}=6$.

Comme $m_{1}, \cdots, m_{6} \geq 1$, on a nécessairement:

$$
m_{1}=m_{2}=m_{5}=m_{6}=1 \text {. }
$$

On tire alors de (1.2.1.2) et $(*)$ :

$$
\begin{aligned}
& 24=242 n_{1}+78 n_{2}+18 n_{3}+48, \\
& 24=6 n_{1}+78 n_{2}+18 n_{3}+48,
\end{aligned}
$$

soit $n_{1}=0$, donc $n_{6}=0$, et (**) $13 n_{2}+3 n_{3}+4=0$. D'autre part, on tire de (1.2.1.2):

$$
24 m_{3}=6 n_{2}+18 n_{3}+48 .
$$

On a donc, utilisant $(* *)$ :

$$
3 n_{2}=1-m_{3} \text {, }
$$

donc

$$
m_{3} \equiv 1(\bmod 3) ; \quad \text { comme } 1 \leq m_{3} \leq 7 \text {, }
$$

$m_{3}$ peut prendre une des trois valeurs 1,4 ou 7 , donc $n_{2}=0,-1$ ou -2 .

Enfin, $(* *)$ entraîne: $n_{2} \equiv 2(\bmod 3)$.

Par conséquent, $n_{2}=-1$, d'où $n_{3}=3$, et $m_{3}=4$. On a ainsi obtenu la solution:

$$
n_{1}=0, n_{2}=-1, n_{3}=3, n_{4}=3, n_{5}=-1, n_{6}=0
$$

du système (i, ii, iii).

On procède de façon analogue pour les autres cas. On constate que la condition (iv) est vérifiée par chacune des solutions de (i, ii, iii). 
1.2.2. Donnons les premiers coefficients des développements en série de Fourier des formes $G_{i}^{(5)}, 1 \leq i \leq 10, i \neq 7$ :

$$
\begin{aligned}
& G_{1}^{(5)} \equiv q+q^{4}+2 q^{7}+2 q^{13}+q^{16}+2 q^{19}+q^{25}-q^{28}-q^{31}-6 q^{34} \\
& \left(\bmod q^{37}\right) \text {; } \\
& G_{2}^{(5)} \equiv q^{4}+q^{7}+2 q^{10}-2 q^{13}-3 q^{19}+q^{22}-5 q^{25}-3 q^{28}+q^{31}+3 q^{34} \\
& \left(\bmod q^{37}\right) \text {; } \\
& G_{3}^{(5)} \equiv q^{7}+q^{10}+2 q^{13}-q^{16}+q^{19}-q^{22}+q^{25}-3 q^{28}-2 q^{31}+q^{34} \\
& \left(\bmod q^{37}\right) \text {; } \\
& G_{4}^{(5)} \equiv q^{4}-2 q^{7}-q^{10}+q^{13}+3 q^{16}+3 q^{19}-5 q^{22}+q^{25}-3 q^{28}-2 q^{31} \\
& \left(\bmod q^{37}\right) \text {; } \\
& G_{5}^{(5)} \equiv q^{7}-2 q^{10}-q^{13}+2 q^{16}+q^{19}+2 q^{22}-2 q^{25}-2 q^{31}-2 q^{34} \\
& \left(\bmod q^{37}\right) \\
& G_{6}^{(5)} \equiv q-2 q^{4}-q^{7}+3 q^{10}-q^{13}+q^{16}+2 q^{19}-3 q^{22}-2 q^{25} \\
& -q^{28}+5 q^{31}+3 q^{34} \quad\left(\bmod q^{37}\right) \text {; } \\
& G_{8}^{(5)} \equiv q^{2}-q^{5}-q^{8}+q^{11}-q^{14}+2 q^{20}-q^{23}-q^{26}-2 q^{29} \\
& +3 q^{32}+4 q^{35} \quad\left(\bmod q^{38}\right) \text {; } \\
& G_{9}^{(5)} \equiv q^{5}-q^{8}-q^{11}-q^{14}+q^{17}+2 q^{20}-q^{23}+2 q^{26}-q^{32}\left(\bmod q^{44}\right) \text {; } \\
& G_{10}^{(5)} \equiv q^{8}-q^{11}-q^{14}+q^{23}+q^{29}-q^{35} \quad\left(\bmod q^{38}\right) \text {. }
\end{aligned}
$$

1.2.3. On démontre comme en 1.1 que ces neuf formes sont orthogonales à $\left\langle\Gamma_{0}\left(3^{4}\right), 2\right\rangle_{0}$ pour le produit scalaire de Petersson (cf. [2], p. 368); plus précisément, cela résulte de ce que la matrice:

$$
\left(\begin{array}{ll}
1 & 0 \\
3^{4} & 1
\end{array}\right)
$$

opère sur la forme parabolique de poids 2 :

$$
\eta(3 z)^{n_{2}} \eta\left(3^{2} z\right)^{n_{3}} \eta\left(3^{3} z\right)^{n_{4}} \eta\left(3^{4} z\right)^{n_{5}}
$$

en la multipliant par

$$
e^{-\left(\left(3 n_{2}+9 n_{3}+3 n_{4}+n_{5}\right) / 12\right) \pi i}
$$

comme il résulte immédiatement de [2], Th. A; or, pour chacune des neuf formes considérées,

$$
3 n_{2}+9 n_{3}+3 n_{4}+n_{5} \equiv \pm 4 \quad(\bmod 24) .
$$

Les neuf formes considérées sont donc des éléments de l'espace engendré 
par les formes primitives dans $\left\langle\Gamma_{0}\left(3^{5}\right), 2\right\rangle_{0}$.

1.2.4. Soient:

$$
\begin{aligned}
G_{7}^{(5)} & =G_{8}^{(5)} \mid T(2), \\
G_{11}^{(5)} & =\frac{1}{3} G_{1}^{(5)} \mid T(2), \\
G_{12}^{(5)} & =G_{2}^{(5)} \mid T(2) .
\end{aligned}
$$

Ces trois formes sont combinaisons linéaires de formes primitives. On a:

$$
\begin{aligned}
& G_{7}^{(5)} \equiv q+q^{4}-q^{7}-q^{13}+q^{16}-q^{19}+q^{25}-4 q^{28}-4 q^{31}+3 q^{34} \\
& \left(\bmod q^{37}\right) \text {; } \\
& G_{11}^{(5)} \equiv q^{2}+q^{8}+q^{14}-2 q^{17}-2 q^{20}-2 q^{23}+q^{26}-2 q^{29}-q^{32}-2 q^{35} \\
& \left(\bmod q^{38}\right) ; \\
& G_{12}^{(5)} \equiv q^{2}+2 q^{5}+2 q^{8}+q^{11}-q^{14}+3 q^{17}-q^{20}+2 q^{23}-4 q^{26} \\
& +4 q^{29}-2 q^{35} \\
& \left(\bmod q^{38}\right) \text {. }
\end{aligned}
$$

L'examen des coefficients de Fourier montre que les douze formes $G_{i}^{(5)}$, $1 \leq i \leq 12$ sont linéairement indépendantes. On peut donc conclure:

Proposition 1.2.5. Les formes $G_{i}^{(5)}, 1 \leq i \leq 12$, constituent une base de l'espace engendré par les formes primitives dans $\left\langle\Gamma_{0}\left(3^{5}\right), 2\right\rangle_{0}$.

\subsection{Diagonalisation}

Pour déterminer les formes primitives elles-mêmes, nous allons utiliser la diagonalisation de $T(2)$. Remarquons tout d'abord que l'espace engendré par les formes $G_{i}^{(5)}, 1 \leq i \leq 12$, est somme directe de l'espace $V$ engendré par $G_{i}^{(5)}, 1 \leq i \leq 7$, et de $W$, engendré par $G_{i}^{(5)}, 8 \leq i \leq 12$, et que $T(2)$ est somme directe de:

$$
T^{\prime}=\left.T(2)\right|_{V}: V \rightarrow W
$$

et

$$
T^{\prime \prime}=\left.T(2)\right|_{W}: W \rightarrow V
$$

qui sont deux opérateurs de rang 5 .

On est donc ramené, pour déterminer les valeurs propres de $T(2)$, à calculer celles de

$$
T^{\prime} \circ T^{\prime \prime}: W \rightarrow W
$$

où $W$ est de dimension 5 . 
L'action de $T(2)$ sur les formes $G_{i}^{(5)}, 1 \leq i \leq 12$, est la suivante:

$$
\begin{aligned}
& G_{1}^{(5)} \mid T(2)=3 G_{11}^{(5)} ; \\
& G_{2}^{(5)} \mid T(2)=G_{12}^{(5)} ; \\
& G_{3}^{(5)} \mid T(2)=G_{9}^{(5)} ; \\
& G_{4}^{(5)} \mid T(2)=G_{8}^{(5)}+6 G_{10}^{(5)} ; \\
& G_{5}^{(5)} \mid T(2)=-2 G_{9}^{(5)} ; \\
& G_{6}^{(5)} \mid T(2)=3 G_{9}^{(5)} ; \\
& G_{7}^{(5)} \mid T(2)=2 G_{8}^{(5)}+3 G_{10}^{(5)}+G_{12}^{(5)} ; \\
& G_{8}^{(5)} \mid T(2)=G_{7}^{(5)} ; \\
& G_{9}^{(5)} \mid T(2)=-\frac{1}{3} G_{1}^{(5)}+G_{3}^{(5)}-G_{5}^{(5)}+\frac{1}{3} G_{6}^{(5)} ; \\
& G_{10}^{(5)} \mid T(2)=\frac{1}{3} G_{2}^{(5)}+\frac{2}{3} G_{4}^{(5)} ; \\
& G_{11}^{(5)} \mid T(2)=\frac{5}{3} G_{1}^{(5)}-2 G_{3}^{(5)}-G_{5}^{(5)}-\frac{2}{3} G_{6}^{(5)} ; \\
& G_{12}^{(5)} \mid T(2)=2 G_{2}^{(5)}+G_{4}^{(5)}+G_{7}^{(5)} .
\end{aligned}
$$

On en déduit lamatrice de $T^{\prime} \circ T^{\prime \prime}$, par rapport à la base $G_{i}^{(5)}, 8 \leq i \leq 12$ :

$$
\left(\begin{array}{rrrrr}
2 & 0 & 3 & 0 & 1 \\
0 & 4 & 0 & -1 & 0 \\
\frac{2}{3} & 0 & 4 & 0 & \frac{1}{3} \\
0 & -2 & 0 & 5 & 0 \\
3 & 0 & 9 & 0 & 3
\end{array}\right) .
$$

Le polynôme caractéristique de cette matrice est:

$$
-(X-3)(X-6)\left(X^{3}-9 X^{2}+18 X-9\right) \text {. }
$$

Soient $\alpha_{1}, \alpha_{2}, \alpha_{3}$ les racines du polynôme:

$$
X^{3}-3 X^{2}+3 \text {. }
$$

On a (cf. [3], § 7):

$$
\alpha_{i}=1+\zeta_{i}+\zeta_{i}^{-1}, \quad(i=1,2,3),
$$

où $\zeta_{i}$ est une racine primitive neuvième de l'unité.

Les valeurs propres de $T^{\prime} \circ T^{\prime \prime}: W \rightarrow W$ sont:

$$
3,6, \alpha_{i}^{2}, \quad i=1,2,3 .
$$

Les valeurs propres de $T(2)$, par conséquent, sont:

$$
0,0, \pm \sqrt{3}, \pm \sqrt{6}, \pm \alpha_{i}, \quad 1 \leq i \leq 3 .
$$


Les espaces propres correspondant à chacune des valeurs propres non nulles sont de dimension 1. L'espace propre associé à la valeur propre 0 est de dimension 2. Il est facile de voir qu'il est engendré par les deux formes primitives normalisées $G_{6}^{(5)}-3 G_{3}^{(5)}$ et $G_{6}^{(5)}+3\left(G_{3}^{(5)}+G_{5}^{(5)}\right)$. On détermine sans peine les autres formes primitives. Le résultat est le suivant:

Proposition 1.3.1. Les formes primitives normalisées de $\left\langle\Gamma_{0}\left(3^{5}\right), 2\right\rangle_{0}$ sont les douze formes $F_{i}^{(5)}, 1 \leq i \leq 12$ :

(a) $F_{1}^{(5)}=G_{6}^{(5)}-3 G_{3}^{(5)}$

$$
\begin{aligned}
& \equiv q-2 q^{4}-4 q^{7}-7 q^{13}+4 q^{16}-q^{19}-5 q^{25}+8 q^{28}+11 q^{31} \\
& \left(\bmod q^{37}\right) \\
& F_{2}^{(5)}=G_{6}^{(5)}+3\left(G_{3}^{(5)}+G_{5}^{(5)}\right) \\
& \equiv q-2 q^{4}+5 q^{7}+2 q^{13}+4 q^{16}+8 q^{19}-5 q^{25}-10 q^{28}-7 q^{31} \\
& \left(\bmod q^{37}\right)
\end{aligned}
$$

(b) $F_{3}^{(5)}=G_{1}^{(5)}-3 G_{5}^{(5)}+\sqrt{3}\left[2 G_{9}^{(5)}+G_{11}^{(5)}\right]$

$$
\begin{aligned}
\equiv & q+\sqrt{3} \cdot q^{2}+q^{4}+2 \sqrt{3} \cdot q^{5}-q^{7}-\sqrt{3} \cdot q^{8}+6 q^{10} \\
& -2 \sqrt{3} \cdot q^{11}+5 q^{13}-\sqrt{3} \cdot q^{14}-5 q^{16}-q^{19}+2 \sqrt{3} \cdot q^{20} \\
& -6 q^{22}-4 \sqrt{3} \cdot q^{23} \\
F_{4}^{(5)}= & G_{1}^{(5)}-3 G_{5}^{(5)}-\sqrt{3}\left[2 G_{9}^{(5)}+G_{11}^{(5)}\right] ;
\end{aligned}
$$

(c) $F_{5}^{(5)}=2 G_{1}^{(5)}-3 G_{3}^{(5)}-G_{6}^{(5)}+\sqrt{6}\left(-G_{9}^{(5)}+G_{11}^{(5)}\right)$, $F_{6}^{(5)}=2 G_{1}^{(5)}-3 G_{3}^{(5)}-G_{6}^{(5)}-\sqrt{6}\left(-G_{9}^{(5)}+G_{11}^{(5)}\right) ;$

(d) $F_{6+i}^{(5)}=\left(\alpha_{i}-1\right)\left(G_{2}^{(5)}+G_{8}^{(5)}\right)+\left(\alpha_{i}^{2}-\alpha_{i}-2\right)\left(G_{4}^{(5)}+3 G_{10}^{(5)}\right)+G_{7}^{(5)}+G_{12}^{(5)}$,

$$
1 \leq i \leq 3 \text {. }
$$

(e) $F_{9+i}^{(5)}=\left(\alpha_{i}-1\right)\left(G_{2}^{(5)}-G_{8}^{(5)}\right)+\left(\alpha_{i}^{2}-\alpha_{i}-2\right)\left(G_{4}^{(5)}-3 G_{10}^{(5)}\right)+G_{7}^{(5)}-G_{12}^{(5)}$

$$
1 \leq i \leq 3 \text {. }
$$

\section{Courbes elliptiques de conducteur une puissance de 3}

\subsection{Conjecture de Weil}

Soit $f(z)$ un élément de $\left\langle\Gamma_{0}\left(3^{\nu}\right), 2\right\rangle_{0}$ qui est fonction propre des opérateurs de Hecke $T(p)$, pour tout $p \neq 3$, et supposons que les valeurs propres associées soient des entiers rationnels. D'après Shimura ([8], Th. 1), il existe alors un morphisme canonique, surjectif, défini $\operatorname{sur} \boldsymbol{Q}$ :

$$
\varphi_{f}: J_{0}\left(3^{\nu}\right) \rightarrow E_{f},
$$

où $E_{f}$ est une courbe elliptique, définie sur $\boldsymbol{Q}$. De plus, la série $L$ de la 
courbe $E_{f}$ coïncide, au facteur local en 3 près, avec la série de Dirichlet associée à $f$.

On a déterminé dans le paragraphe 1 les formes primitives de poids 2 normalisées sur $\Gamma_{0}\left(3^{\nu}\right)$, pour $\nu=3,4,5$ resp. Trois d'entre elles ont des coefficients entiers rationnels. Ce sont:

$F_{1}^{(3)}$, qui est l'unique forme primitive normalisée de poids 2 sur $\Gamma_{0}\left(3^{3}\right)$;

$F_{1}^{(5)}, F_{2}^{(5)}$, qui sont primitives sur $\Gamma_{0}\left(3^{5}\right)$.

Considérons les trois courbes elliptiques associées. Ces courbes ne sont pas isogènes $\operatorname{sur} \boldsymbol{Q}$, puisque leurs séries $L$ sont distinctes. En outre, chacune d'entre elles a bonne réduction en dehors de 3 .

D'autre part, on connait toutes les courbes elliptiques définies sur $\boldsymbol{Q}$ qui ont bonne réduction en dehors de 3, cf. [1], appendice. Ces courbes se partagent en trois classes de $\boldsymbol{Q}$-isogénie; 1'une de ces classes a pour conducteur $3^{3}$, les deux autres ont pour conducteur $3^{5}$; en particulier, le facteur local de la fonction $L$ en 3 est 1 pour chacune des trois classes.

La comparaison de ces renseignements montre que les trois courbes associées à $F_{1}^{(3)}$ d'une part, $F_{1}^{(5)}$ et $F_{2}^{(5)}$ d'autre part, représentent les trois classes de $\boldsymbol{Q}$-isogénie en question. L'examen des premiers coefficients du développement de Fourier permet de reconnaître la classe correspondant à $F_{1}^{(5)}$ et celle correspondant à $F_{2}^{(5)}$.

On a ainsi démontré:

THÉORÈme 2.1.1 (Conjecture de Weil pour les courbes de conducteur une puissance de 3$)$. Soit $L(s)=\sum_{n=1}^{\infty} a_{n} \cdot n^{-s}$ la fonction $L$ d'une courbe elliptique définie sur $\mathbf{Q}$, de conducteur $3^{\nu}($ donc $\nu=3$ ou 5$)$. Alors la série de Fourier:

$$
f(z)=\sum_{n=1}^{\infty} a_{n} \cdot q^{n}
$$

est une forme primitive normalisée sur $\left\langle\Gamma_{0}\left(3^{\nu}\right), 2\right\rangle_{0}$, donc coïncide avec $F_{1}^{(3)}$ si $\nu=3$, avec $F_{1}^{(5)}$ ou $F_{2}^{(5)}$ si $\nu=5$. De plus, il existe un $\mathbf{Q}$-morphisme surjectif de $J_{0}\left(3^{\nu}\right)$ sur la courbe considérée.

La courbe associée à $F_{1}^{(3)}$ est la courbe $X_{0}(27)$, d'équation:

$$
y^{2}+y=x^{3}-7
$$

(cf. [1], table p. 209).

La classe d'isogénie correspondant à $F_{1}^{(5)}$, resp. $F_{2}^{(5)}$ est représentée par la courbe: 


$$
y^{2}+y=x^{3}+20
$$

resp.

(loc. cit).

$$
y^{2}+y=x^{3}+2
$$

2.2. Multiplications complexes

Considérons les endomorphismes $R_{3, \pm}^{*}$ de $\left\langle\Gamma_{0}\left(3^{\nu}\right), 2\right\rangle_{0}, \nu=3,4,5$, définis par:

$$
R_{3, \pm}^{*}: f(z) \mapsto f\left(z \pm \frac{1}{3}\right) .
$$

Soit $R_{3}^{*}=R_{3,+}^{*}-R_{3,-}^{*}$.

Lorsque $f(z)$ est une forme primitive normalisée:

$$
f \mid R_{3}^{*}=i \sqrt{3} \cdot f_{x},
$$

où $f_{z}$ note la forme tordue par le caractère de Legendre modulo 3 (cf. [5], I.3.4).

L'espace $\left\langle\Gamma_{0}\left(3^{\nu}\right), 2\right\rangle_{0}$ s'identifie de façon canonique à l'espace cotangent à l'origine à la variété $J_{0}\left(3^{\nu}\right)$. Les opérateurs $R_{3}^{*}, R_{3, \pm}^{*}$ sont induits par des endomorphismes $R_{3}, R_{3, \pm}$ de $J_{0}\left(3^{\nu}\right)$; ces endomorphismes sont définis $\operatorname{sur} Q(\sqrt{-3})$ (cf. [8], §4; [5], I, 3.4).

Soit $f$ l'une des trois formes primitives $F_{1}^{(3)}, F_{1}^{(5)}, F_{2}^{(5)}$; soit $E_{f}$ la courbe elliptique associée à $f$ (cf. [8], Prop. 3). Soit $R$ l'un des endomorphismes $R_{3}, R_{3, \pm}$. Il existe un endomorphisme de la courbe $E_{f}$, noté encore $R$, qui rend commutatif le diagramme suivant:

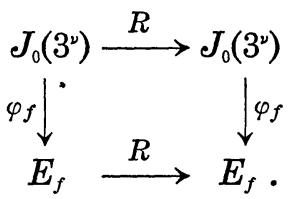

L'anneau des endomorphismes de $E_{f}$ contient donc $R_{3}, R_{3, \pm}$, qui vérifient:

$$
R_{3}^{2}=-3 ; \quad R_{3,+}+R_{3,-}=-1 ; \quad R_{3,+}-R_{3,-}=R_{3} \text {. }
$$

Par conséquent, la courbe $E_{f}$ admet une multiplication complexe par l'anneau des entiers de $\boldsymbol{Q}(\sqrt{-3})$ :

Proposition 2.2.1. Soit $f$ l'une des trois formes primitives $F_{1}^{(3)}, F_{1}^{(5)}$, $F_{2}^{(5)}$. La courbe $E_{f}$ associée à $f$ admet une multiplication complexe par 
l'anneau des entiers de $Q(\sqrt{-3})$. En particulier, son invariant est nul.

Soit toujours $f$ l'une des formes $F_{1}^{(3)}, F_{1}^{(5)}, F_{2}^{(5)}$, et considérons la courbe $E_{f}$. D'après Deuring, et la Prop. 2.2.1, la série $L$ de $E_{f}$ est associée à un Grössencharakter. Montrons comment cette remarque permet de déterminer a priori les formes $F_{1}^{(3)}, F_{1}^{(5)}$ et $F_{2}^{(5)}$.

Utilisons les notations de Shimura [7]. Soit $\omega=e^{2 \pi i / 3}$, et $K=\boldsymbol{Q}(\omega)$. Soit $\boldsymbol{m}$ un idéal entier de $K$, et $\lambda$ un Grössencharakter modulo $\boldsymbol{m}$. La série $L$ associée à $\lambda$ est par définition:

$$
L(\lambda, s)=\sum_{\mathfrak{a}} \lambda(\mathfrak{a}) \cdot N(\mathfrak{a})^{-s},
$$

a parcourant les idéaux entiers de $K$ premiers à $\boldsymbol{m}$.

Le lemme 3 de [7] montre que les Grössencharaktere susceptibles de correspondre à des formes de poids 2 sont ceux qui vérifient:

$$
\lambda((\alpha))=\alpha
$$

pour tout $\alpha \in K^{\times}$tel que $\alpha \equiv 1 \bmod ^{\times} \boldsymbol{m}$.

On note:

$$
f_{\lambda}(z)=\sum_{a} \lambda(\mathfrak{a}) \cdot e^{2 \pi i N(\mathfrak{a}) z},
$$

a parcourant les idéaux entiers de $K$ premiers à $\boldsymbol{m}$. On dit que $f_{\lambda}$ est la forme associée à $\lambda$. Enfin, on associe à $\lambda$ un caractère de Dirichlet modulo $3 \cdot N(\boldsymbol{m})$ :

$$
\varepsilon_{\lambda}(a)=\left(\frac{-3}{a}\right) \cdot \frac{\lambda((a))}{a},
$$

pour tout $a \in Z$ premier à $\boldsymbol{m}$.

LEMME 2.2.3. a) Il existe un unique Grössencharakter $\lambda_{1}^{(3)}$ de $K$ de conducteur 3 , et vérifiant (2.2.2).

b) Il existe exactement deux Grössencharaktere $\lambda_{1}^{(5)}$ et $\lambda_{2}^{(5)}$ de $K$, de conducteur 9, vérifiant (2.2.2), et tels que le caractère de Dirichlet associé soit le caractère unité. On $a$ :

$$
\lambda_{i}^{(5)}((1+3 \omega))=\omega^{i}, \quad i=1,2 .
$$

Démonstration. Soit $\alpha$ un entier de $K$ premier à 3 . Alors un et un seul des trois nombres $\pm \alpha, \pm \omega \alpha, \pm \omega^{2} \alpha$ est congru à $1(\mathrm{mod}) 3$, et $\lambda_{1}((\alpha))$ est le nombre en question; d'où (a). 
Soit $\alpha$ un entier de $K$, congru à $1(\bmod 3)$. Alors un et un seul des neuf nombres $4^{i}(1+3 \omega)^{j} \alpha, 0 \leq i, j \leq 2$ est congru à $1(\bmod 9)$. Par conséquent, la connaissance d'un Grössencharakter $(\bmod 9)$ est déterminée par celle de $\lambda((4))$ et $\lambda((1+3 \omega))$, qui sont nécessairement des racines cubiques de l'unité. La condition sur $\varepsilon_{\lambda}$ entraîne que $\lambda((4))=1$. Les possibilités $\lambda((1+3 \omega))=1, \omega, \omega^{2}$ resp. donnent $\lambda_{1}^{(3)}, \lambda_{1}^{(5)}, \lambda_{2}^{(5)}$ resp.

Nous pouvons maintenant utiliser le Lemme 3 de [7]. Ce dernier montre que $F_{1}^{(3)}$ est un élément normalisé de $\left\langle\Gamma_{0}\left(3^{3}\right), 2\right\rangle_{0}$, et $F_{1}^{(5)}, F_{2}^{(5)}$ des éléments normalisés de $\left\langle\Gamma_{0}\left(3^{5}\right), 2\right\rangle_{0}$; on vérifie sans peine:

Proposition 2.2.4. Les formes $F_{1}^{(3)}, F_{1}^{(5)}, F_{2}^{(5)}$ resp. sont associees aux Grössencharaktere $\lambda_{1}^{(3)}, \lambda_{1}^{(5)}, \lambda_{2}^{(5)}$ resp.

\section{Description de $J_{0}\left(3^{4}\right)$}

Le but de ce paragraphe est la description des courbes elliptiques qui sont des quotients de $J_{0}\left(3^{4}\right)$ en termes de réseaux complexes. La technique employée pour ce faire est celle décrite dans [5], I, §4. Nons nous contenterons ici de rappeler brièvement les notations utilisées, en renvoyant à loc. cit. pour plus de détails.

\subsection{Détermination des réseaux}

Soit $\mathscr{H}\left(3^{4}\right)$ le groupe défini par [5], I, Prop. 3.2.3. Un premier calcul, élémentaire, permet de montrer:

LEMME 3.1.1. Le groupe $\mathscr{H}\left(3^{4}\right)$ admet pour base sur $Z$ les huit éléments suivants:

$$
(\tilde{c}: \tilde{1}), \quad c \in \mathscr{B},
$$

où $\mathscr{B}=\{2,4,7,13,25,31,34,58\}$.

L'expression de ( $\tilde{c}: \tilde{1}), 1 \leq c<3^{4},(c, 3)=1$, en fonction de cette base est donnée par la table 1.

Posons:

$$
\left\{\begin{array}{l}
f_{1}=F_{1}^{(3)} ; \\
f_{2}=F_{1}^{(3)}(z)-3 F_{1}^{(3)}(3 z) ; \\
f_{3}=F_{1}^{(4)} ; \\
f_{4}=F_{2}^{(4)} ;
\end{array}\right.
$$


(cf. 1.1.1).

Les formes $f_{i}, 1 \leq i \leq 4$ constituent une base de l'espace $\left\langle\Gamma_{0}\left(3^{4}\right), 2\right\rangle_{0}$; de plus, ce sont des vecteurs propres normalisés des opérateurs de Hecke; enfin, $f_{3}$ et $f_{4}$ sont primitives.

Soient $z_{0}, z_{1} \in \mathfrak{S}^{*}$. On note:

$$
\left\{z_{0}, z_{1}\right\}
$$

le vecteur de $C^{4}$ de coordonnées:

$$
\left\{z_{0}, z_{1}\right\}_{f_{i}}=\int_{z_{0}}^{z_{1}} f_{i}(t) d t, \quad(1 \leq i \leq 4) .
$$

L'application

$$
\xi: \mathscr{H}\left(3^{4}\right) \rightarrow C^{4}
$$

définie par:

$$
\xi((\tilde{c}: \tilde{d}))=\left\{\frac{b}{d}, \frac{a}{c}\right\},
$$

où $a, b, c, d \in Z$ vérifient $a d-b c=1$, et $\tilde{c} \equiv c\left(\bmod 3^{4}\right), \tilde{d} \equiv d\left(\bmod 3^{4}\right)$, définit un isomorphisme

$$
\xi: \mathscr{H}\left(3^{4}\right) \rightarrow L
$$

où $L$ est un réseau de $C^{4}$.

D'après Shimura, cf. [8], $\S 3$, les points de la jacobienne $J=J_{0}\left(3^{4}\right)$ à valeurs dans $C$ s'identifient au quotient $C^{4} / L$. L'application:

$$
\begin{aligned}
\varphi: \mathfrak{S}^{*} & \rightarrow C^{4} \\
z & \mapsto\{0, z\}
\end{aligned}
$$

définit par passage au quotient un plongement de $X_{0}\left(3^{4}\right)(C) \simeq \mathscr{S C}^{*} / \Gamma_{0}\left(3^{4}\right)$ dans la jacobienne $J(C) \simeq C^{4} / L$; ce plongement envoie la pointe $z=0$ de $X_{0}\left(3^{4}\right)$ sur l'origine de $J$.

Les formes $f_{1}, f_{2}$ ont des coefficients entiers rationnels; ceux de $f_{3}$ sont des entiers du corps $Q(\sqrt{3})$, et ceux de $f_{4}$ se déduisent des précédents par conjugaison. D'après Shimura [8], Prop. 3, il existe donc trois morphismes canoniques, définis sur $\boldsymbol{Q}$, et surjectifs:

$$
\begin{gathered}
\varphi_{1}: J \rightarrow E_{1}, \\
\varphi_{2}: J \rightarrow E_{2}, \\
\varphi_{A}: J \rightarrow A,
\end{gathered}
$$


où $E_{1}, E_{2}$ sont des courbes elliptiques définies $\operatorname{sur} Q$, et $A$ une variété abélienne de dimension 2 , définie sur $\boldsymbol{Q}$.

Soient $\pi_{1}, \pi_{2}, \pi_{A}$ les projections canoniques de $C^{4}$ sur $C, C, C^{2}$ correspondant à l'isomorphisme:

$$
C^{4} \simeq C \times C \times C^{2}
$$

Posons

$$
L_{i}=\pi_{i}(L), \quad i=1,2
$$

et

$$
L_{A}=\pi_{A}(L) .
$$

Si l'on identifie $J(C)$ à $C^{4} / L$, les morphismes, $\varphi_{i}, \varphi_{A}, \mathrm{i}=1,2$, s'obtiennent à partir de $\pi_{i}, \pi_{A}$ par passage au quotient.

La conjugaison complexe opère sur $L$ (cf. [5], I, Prop. 3.2.5). On note $L^{ \pm}$les sous-réseaux de $L$ correspondant aux valeurs propres \pm 1 .

En termes des générateurs choisis au Lemme 3.1.1, la conjugaison complexe se traduit par les formules de la table 2. On en déduit:

Lemme 3.1.2. Le réseau $L^{ \pm}$est engendré par les vecteurs:

$$
\begin{aligned}
& \gamma_{1}^{ \pm}=\left\{0, \frac{1}{4}\right\} \pm\left\{0,-\frac{1}{4}\right\} ; \\
& \gamma_{2}^{ \pm}=\left\{0, \frac{1}{7}\right\} \pm\left\{0,-\frac{1}{7}\right\} ; \\
& \gamma_{3}^{ \pm}=\left\{0, \frac{1}{13}\right\} \pm\left\{0,-\frac{1}{13}\right\} ; \\
& \gamma_{4}^{ \pm}=\left\{0, \frac{1}{31}\right\} \pm\left\{0,-\frac{1}{31}\right\} .
\end{aligned}
$$

La table 3 donne l'expression de $\gamma_{k}^{ \pm}, k=1, \cdots, 4$, en fonction des générateurs définis en 3.1.1, et inversement.

L'examen de la table 3 montre en particulier:

Proposition 3.1.3. Le réseau $L$ est un sous-réseau d'indice $2^{4} d u$ réseau $\frac{1}{2} L^{+} \oplus \frac{1}{2} L^{-}$. Plus précisément, soit

$$
z=\frac{1}{2} \sum_{k=1}^{4}\left(x_{k} \gamma_{k}^{+}+y_{k} \gamma_{k}^{-}\right), \quad x_{k}, y_{k} \in Z,
$$

un élément $d u \quad$ réseau $\frac{1}{2} L^{+} \oplus \frac{1}{2} L^{-}$. Alors

$$
z \in L
$$


si et seulement si $x_{k} \equiv y_{k}(\bmod 2), 1 \leq k \leq 4$.

3.1.4. Notons $\underline{c}$ la matrice diagonale qui représente l'action de $T(2)$ sur la base $\left\{f_{1}, \cdots, f_{4}\right\}$ de l'espace $\left\langle\Gamma_{0}\left(3^{4}\right), 2\right\rangle_{0}$ :

$$
\underline{c}=\operatorname{diag}\left(c_{1}, \cdots, c_{4}\right) \text {. }
$$

(On sait, du reste, que $\underline{c}=\operatorname{diag}(0,0, \sqrt{3},-\sqrt{3})$, cf. 1.1).

L'action de $T(2)$ se traduit par la formule:

$$
\underline{c} \cdot\{0, z\}=\{0,2 z\}+\left\{0, \frac{z+1}{2}\right\}+\left\{0, \frac{z}{2}\right\}-\left\{0, \frac{1}{2}\right\},
$$

pour tout $z \in \mathfrak{S}^{*}$; cf. [5].

Notons $\gamma_{k, i}^{ \pm}$la $i$-ième coordonnée de $\gamma_{k}^{ \pm}, 1 \leq i, k \leq 4$.

Procédant comme dans [5], I, 4.9, on écrit la formule (3.1.4.1), pour $\pm z=\frac{1}{4}, \frac{1}{7}, \frac{1}{13}, \frac{1}{31}$. On obtient ainsi, séparant les parties correspondant aux valeurs +1 et -1 de la conjugaison complexe, les deux systèmes suivants de quatre équations aux inconnues $\gamma_{k, i}^{ \pm}$:

$$
\left\{\begin{array}{l}
\left(c_{i}-2\right) \gamma_{1, i}^{+}+2 \gamma_{2, i}^{+}-2 \gamma_{3, i}^{+}=0 ; \\
-\gamma_{1, i}^{+}+\left(c_{i}+1\right) \gamma_{2, i}^{+}-\gamma_{3, i}^{+}=0 ; \\
-\gamma_{2, i}^{+}+\left(c_{i}+1\right) \gamma_{3, i}^{+}+\gamma_{4, i}^{+}=0 ; \\
\gamma_{1, i}^{+}-\gamma_{2, i}^{+}+\gamma_{3, i}^{+}+c_{i} \cdot \gamma_{4, i}^{+}=0 .
\end{array}\right.
$$

resp.

$$
\left\{\begin{array}{l}
c_{i} \cdot \gamma_{1, i}^{-}=0 ; \\
-\gamma_{1, i}^{-}+\left(c_{i}+1\right) \gamma_{2, i}^{-}-\gamma_{3, i}^{-}=0 ; \\
-\gamma_{2, i}^{-}+\left(c_{i}-1\right) \gamma_{3, i}^{-}+\gamma_{4, i}^{-}=0 ; \\
\gamma_{1, i}^{-}-\gamma_{2, i}^{-}+\gamma_{3, i}^{-}+c_{i} \cdot \gamma_{4, i}^{-}=0 .
\end{array}\right.
$$

Le système (3.1.4.2) resp. (3.1.4.3) n'a de solutions non triviales que pour $c_{i} \in\{0, \pm \sqrt{3}\}$. Pour $c_{i}= \pm \sqrt{3}$, le système est de rang 3 , ce qui détermine $\gamma_{k, i}^{+}$resp. $\gamma_{\bar{k}, i}$ à une constante multiplicative près.

Lorsque $c_{i}=0$, les systèmes considérés sont de rang 2. On doit alors utiliser l'identité:

$$
f_{2}(z)=f_{1}(z)-3 f_{1}(3 z), \quad \text { cf. }(3.1 .1 .1)
$$

qui entraîne:

$$
\{0, z\}_{f_{2}}=\{0, z\}_{f_{1}}-\{0,3 z\}_{f_{1}}
$$


Les résultats obtenus sont rassemblés dans la table 4 . On a posé

$$
\begin{aligned}
& \omega_{1}^{+}=\gamma_{1,1}^{+} ; \omega_{2}^{+}=-3 \omega_{1}^{+} ; \omega_{3}^{+}=\gamma_{2,3}^{+} ; \omega_{4}^{+}=\gamma_{2,4}^{+} ; \\
& \omega_{1}^{-}=\gamma_{1,1}^{-} ; \omega_{2}^{-}=\gamma_{1,1}^{-} ; \omega_{3}^{-}=\gamma_{2,3}^{-} ; \omega_{4}^{-}=\gamma_{2,4}^{-} .
\end{aligned}
$$

\subsection{Pointes de $X_{0}\left(3^{4}\right)$}

Soit $z$ une pointe de $X_{0}\left(3^{4}\right)$. Pour calculer $\{0, z\}$, nous procéderons comme dans [5], I, 4.10. Plus précisément, on utilise la formule (3.1.4.1), ainsi que [5], Prop. 3.2.4.

Montrons comment se fait le calcul pour la pointe à l'infini. La formule (3.1.4.1) s'écrit:

$$
(\underline{c}-3) \cdot\{0, \infty\}=-\left\{0, \frac{1}{2}\right\}=-\gamma_{1}^{+}+\gamma_{2}^{+}-\gamma_{3}^{+}-\gamma_{4}^{+}
$$

(en utilisant la table 3).

D'après la table 4 , on a donc:

$$
\begin{aligned}
& \{0, \infty\}_{f_{1}}=\frac{1}{3} \omega_{1}^{+} ; \\
& \{0, \infty\}_{f_{2}}=0 ; \\
& \{0, \infty\}_{f_{3}}=(\sqrt{3} / 3) \omega_{3}^{+} ; \\
& \{0, \infty\}_{f_{4}}=-(\sqrt{3} / 3) \omega_{4}^{+} .
\end{aligned}
$$

Les résultats obtenus sont rassemblés dans la table 5, où l'on a posé:

$$
\{0, \pm z\}=\frac{1}{2}\left[X(z, k) \cdot \omega_{k}^{+} \pm Y(z, k) \cdot \omega_{k}^{-}\right], \quad 1 \leq k \leq 4 .
$$

3.3. Utilisation de $R_{3}^{*}$

Considérons à nouveau l'opérateur $R_{3}^{*}$, cf. 2.2. On a:

$$
\begin{aligned}
& f_{1} \mid R_{3}^{*}=i \sqrt{3} \cdot f_{1}=i \sqrt{3} \cdot f_{1, \chi} ; \\
& f_{3} \mid R_{3}^{*}=i \sqrt{3} \cdot f_{4}=i \sqrt{3} \cdot f_{3, \chi} ; \\
& f_{4} \mid R_{3}^{*}=i \sqrt{3} \cdot f_{3}=i \sqrt{3} \cdot f_{4, \chi} .
\end{aligned}
$$

(On note $f_{x}$ la forme tordue de $f$ par le caractère de Legendre modulo 3 ).

Appliquons à $f_{1}, f_{3}, f_{4}$ l'analogue de [5], I, Lemme 4.11.3.1. On a:

$$
\{0, z\}_{f_{\chi}}=-\frac{i \sqrt{3}}{3}\left[\left\{\frac{1}{3}, z+\frac{1}{3}\right\}_{f}-\left\{\frac{2}{3}, z+\frac{2}{3}\right\}_{f}\right] .
$$

Faisons $z=\infty$ dans cette formule; compte tenu des résultats de la table 5 , ceci donne, pour $f_{1}$ : 


$$
\omega_{1}^{+}=\frac{i \sqrt{3}}{3} \omega_{1}^{-}
$$

et pour $f_{3}, f_{4}$ :

$$
\left\{\begin{array}{l}
\omega_{4}^{+}=-\frac{i \sqrt{3}}{3} \cdot \omega_{3}^{-} ; \\
\omega_{3}^{+}=-\frac{i \sqrt{3}}{3} \cdot \omega_{4}^{-} .
\end{array}\right.
$$

La Prop. 3.1.3, et le fait que $L$ soit un réseau dans $C^{4}$, entraînent que tout vecteur $z=\left(z_{1}, \cdots, z_{4}\right)$ de $C^{4}$ s'écrit de façon unique:

$$
z=\frac{1}{2} \sum_{k=1}^{4}\left(x_{k} \gamma_{k}^{+}+y_{k} \gamma_{k}^{-}\right), \quad \text { où } x_{k}, y_{k} \in R \text {. }
$$

Soient $L_{i}=\pi_{i}(L), i=1,2$. Soient:

$$
z_{i}=\frac{1}{2}\left(a_{i} \omega_{i}^{+}+b_{i} \omega_{i}^{-}\right), \quad i=1,2 .
$$

Tout vecteur de $C$ s'écrit de façon unique sous cette forme, avec $a_{i}$, $b_{i} \in R$.

Soit enfin $L_{A}=\pi_{A}(L)$. Notons:

$$
M_{k}(a, b, c, d)=\frac{1}{2}\left[a \omega_{k}^{+}+b \omega_{k}^{-}+\sqrt{3}\left(c \omega_{k}^{+}+d \omega_{k}^{-}\right)\right], \quad k=3,4 .
$$

Proposition 3.3.4. a) Pour que $z_{i} \in C$ soit un élément $d u$ réseau $L_{i}$ $(i=1,2)$, il faut et il suffit qu'il existe $a_{i}, b_{i} \in Z, a_{i} \equiv b_{i}(\bmod 2)$, tels que

$$
z_{i}=\frac{1}{2}\left(a_{i} \omega_{i}^{+}+b_{i} \omega_{i}^{-}\right) .
$$

b) Pour que $\left(z_{3}, z_{4}\right) \in C^{2}$ soit un élément $d u$ réseau $L_{A}$, il faut et il suffit qu'il existe $a, b, c, d \in Z, a \equiv b(\bmod 2), c \equiv d(\bmod 2)$, tels que:

$$
\left(z_{3}, z_{4}\right)=\left(M_{3}(a, b, c, d), M_{4}(a, b,-c,-d)\right) .
$$

Démonstration. Soit $z \in C^{4}$ un vecteur de $L$, que nous écrivons:

$$
z=\frac{1}{2} \sum_{k=1}^{4}\left(x_{k} \gamma_{k}^{+}+y_{k} \gamma_{k}^{-}\right), \quad x_{k}, y_{k} \in Z, \quad x_{k} \equiv y_{k}(\bmod 2) \text {. }
$$

On a donc:

$$
z_{i}=\frac{1}{2} \sum_{k=1}^{4}\left(x_{k} \gamma_{k, i}^{+}+y_{k} \gamma_{k, i}^{-}\right), \quad 1 \leq i \leq 4 .
$$

Tirant de la table 4 les valeurs de $\gamma_{k, i}^{ \pm}$en fonction de $\omega_{i}^{ \pm}$, et tenant compte des identités (3.3.2), on obtient la nécessité des conditions de la proposition. 
On a porté dans la table 6 l'expression de $a_{i}, b_{i}(i=1,2), a, b, c, d$, en fonction de $x_{k}, y_{k}, 1 \leq k \leq 4$, et réciproquement. Inversement, il est clair que $z_{i}=\frac{1}{2}\left(a_{i} \omega_{1}^{+}+b_{i} \omega_{1}^{-}\right), i=1,2, a_{i}, b_{i} \in Z, a_{i} \equiv b_{i}(\bmod 2)$, est la $i$-ième projection du vecteur $\frac{1}{2}\left(a_{i} \gamma_{i}^{+}+b_{i} \gamma_{i}^{-}\right) \in L$.

Enfin $\left(M_{3}(a, b, c, d), M_{4}(a, b,-c,-d)\right)$ est l'image par $\pi_{A}$ du vecteur $\frac{1}{2}\left(a \gamma_{2}^{+}+b \gamma_{2}^{-}+c \gamma_{3}^{+}+d \gamma_{3}^{-}\right)$qui est un élément de $L$; d'où la suffisance.

Corollaire. Tout vecteur de $C^{2}$ s'écrit de façon unique

$$
\left(z_{3}, z_{4}\right)=\left(M_{3}(a, b, c, d), M_{4}(a, b,-c,-d)\right),
$$

où $a, b, c, d \in \boldsymbol{R}$.

Nous allons également tirer de la table 6 le résultat suivant:

Proposition 3.3.5. Le noyau du morphisme canonique

$$
J \rightarrow E_{1} \times E_{2} \times A
$$

est produit de deux groupes cycliques d'ordre 3. Plus précisément, identifiant les points de $J$ au quotient de $C^{4}$ par $L$, ce noyau est engendré par les vecteurs:

$$
\frac{1}{3}\left(\gamma_{1}^{+}-\gamma_{4}^{+}\right)+\frac{1}{3}\left(\gamma_{1}^{-}-2 \gamma_{2}^{-}-\gamma_{4}^{-}\right)
$$

et

$$
\frac{2}{3}\left(\gamma_{1}^{-}+\gamma_{2}^{-}+\gamma_{4}^{-}\right) \quad d e C^{4} .
$$

Démonstration. Il résulte immédiatement de l'examen de la table 6 que le noyau considéré est annulé par la multiplication par 3. C'est donc un espace vectoriel sur $F_{3}$, de dimension égale au rang du système de la table 6 ( $\operatorname{sur} F_{3}$ ); ce rang est 2 ; le calcul explicite donne aisément le résultat annoncé.

Il est commode d'exprimer les coordonnées des pointes de $X_{0}\left(3^{4}\right)$ en fonction de $a_{i}, b_{i}, i=1,2, a, b, c, d$; ceci est fait dans la table 7 .

\section{Applications}

4.1. Courbes elliptiques de conducteur 27

On sait (par ex. [1], appendice) qu'il existe quatre courbes elliptiques définies sur $\boldsymbol{Q}$, de conducteur 27, et que ces courbes sont $\boldsymbol{Q}$-isogènes entre elles. Nous allons retrouver l'existence de ces courbes et des isogénies qui les relient en utilisant les résultats du paragraphe 3. 
4.1.1. Rappelons quelle est l'action du groupe de Galois $G_{\boldsymbol{Q}}=\operatorname{Gal}(\overline{\boldsymbol{Q}} / \boldsymbol{Q})$ sur les pointes de la courbe $X_{0}\left(3^{4}\right)$; toutes ces pointes sont rationnelles sur le corps des racines neuvièmes de l'unité $\boldsymbol{Q}\left(e^{2 y i / 9}\right)=K^{\prime}$. Soit:

$$
\begin{aligned}
& n \mapsto[n] \\
&(Z / 9 Z)^{\times} \rightarrow \operatorname{Gal}\left(K^{\prime} / Q\right)=G
\end{aligned}
$$

l'isomorphisme canonique. Alors $G$ est engendré par l'élément [2]. Si $z=a / d,\left(a \in Z, d\right.$ divise $\left.3^{4}\right)$ représente une pointe de $X_{0}\left(3^{4}\right)$, la transformée par [2] de cette pointe est représentée par $z^{\prime}=5 a / d$ (cf. [5], II, §3). En particulier, [2] laisse stables les pointes 0 et $\infty$, et échange $\pm 1 / 3$, resp. $\pm 1 / 27$.

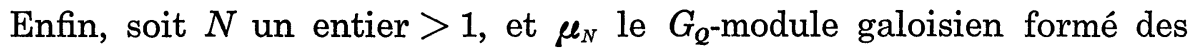
racines $N$-ièmes de l'unité. On note $\mu_{N}^{\otimes k}$ le produit tensoriel de $k$ copies de $\boldsymbol{\mu}_{N}$, muni de sa structure de $G_{\boldsymbol{Q}}$-module. Cette notation ne dépend que de $k \bmod \varphi(N)$. En particulier, on note $Z / N Z=\mu_{N}^{\otimes 0} ;$ cf. [5], I, 2.5.

4.1.2. Considérons les courbes $E_{1}, E_{2}$ associées aux formes $f_{1}, f_{2}$, cf. 3.1.1.1. Ces deux courbes elliptiques sont de conducteur 27, la courbe $E_{1}$ n'étant rien d'autre que la courbe modulaire $X_{0}(27)$.

Soient $C_{1}, C_{2}$ resp. les sous-groupes engendrés dans $E_{1}$, resp. $E_{2}$ par les images des pointes de $X_{0}\left(3^{4}\right)$; le groupe $G$ permute les pointes, et fait ainsi de $C_{1}, C_{2}$ des $G_{Q}$-modules galoisiens. Le lemme suivant détermine leur structure:

LEMme 4.1.2.1.

$$
C_{1} \cong Z / 3 Z \oplus \mu_{3} ; \quad C_{2} \cong \mu_{9}^{\otimes 2} \oplus \mu_{3}
$$

Démonstration. Posons $\omega_{i}^{+} / \omega_{i}^{-}=2 \tau_{i}-1, i=1,2$. D'après la Prop. 3.3.4, a), les points de $E_{i}$ à valeurs dans $C$ s'identifient au quotient $C / L_{i}$, où

$$
L_{i}=\omega_{i}^{-} \cdot\left[1, \tau_{i}\right], \quad i=1,2 .
$$

La table 7 montre que les coordonnées des images des pointes dans le parallélogramme fondamental du réseau $\left[1, \tau_{i}\right]$, construit sur $1, \tau_{i}$, sont les suivantes: 


\begin{tabular}{|c|c|}
\hline pointes & réseau $\left[1, \tau_{1}\right]$ \\
\hline 0 & $(0,0)$ \\
\hline$\infty, \pm \frac{1}{27}$ & $\left(\frac{2}{3}, \frac{2}{3}\right)$ \\
\hline$\frac{1}{3}$ & $\left(\frac{2}{3}, 0\right)$ \\
\hline$-\frac{1}{3}$ & $\left(\frac{1}{3}, 0\right)$ \\
\hline$\frac{1}{9}, \frac{4}{9}, \frac{7}{9}$ & $\left(\frac{1}{3}, \frac{2}{3}\right)$ \\
\hline$-\frac{1}{9}-\frac{4}{9}-\frac{7}{9}$ & $\left(0, \frac{2}{3}\right)$ \\
\hline
\end{tabular}

\begin{tabular}{|c|c|}
\hline pointes & réseau $\left[1, \tau_{2}\right]$ \\
\hline $0, \infty$ & $(0,0)$ \\
\hline$\frac{1}{3},-\frac{1}{27}$ & $\left(\frac{1}{3}, \frac{2}{3}\right)$ \\
\hline$-\frac{1}{3}, \frac{1}{27}$ & $\left(0, \frac{2}{3}\right)$ \\
\hline$\pm \frac{1}{9}$ & $\left(\frac{7}{9}, \frac{4}{9}\right)$ \\
\hline$\pm \frac{2}{9}$ & $\left(\frac{4}{9}, \frac{1}{9}\right)$ \\
\hline$\pm \frac{4}{9}$ & $\left(\frac{1}{9}, \frac{7}{9}\right)$ \\
\hline
\end{tabular}

L'image de $\infty$ engendre dans $E_{1}$ un groupe d'ordre 3 formé de points $Q$-rationnels, donc isomorphe à $Z / 3 Z$.

L'image de $\frac{1}{3}$ engendre dans $E_{1}$ un groupe d'ordre 3 formé de points rationnels sur le corps $\boldsymbol{Q}(\omega)$ des racines cubiques de l'unité; l'élément nontrivial du groupe de Galois permute les images des pointes $\frac{1}{3}$ et $-\frac{1}{3}$; d'où un $\mathrm{G}_{Q^{-}}$-module isomorphe à $\mu_{3}$. Enfin, la somme directe des deux groupes considérés contient les images de toutes les pointes.

Soient $P, P^{\prime}$ les images dans $E_{2}$ des pointes $\frac{1}{9}, \frac{1}{3}$. Les images dans $E_{2}$ des pointes $-\frac{1}{3}, \frac{2}{9}, \frac{4}{9}$ resp. sont $3 P+2 P^{\prime}, 7 P, 4 P$ resp. Soit

$$
Q=3 P+P^{\prime} \text {. }
$$

On a:

$$
\begin{aligned}
& P^{[2]}=4 P ; \\
& Q^{[2]}=-Q .
\end{aligned}
$$

Ceci montre: le groupe engendré par $P$ est isomorphe à $\mu_{9}^{\otimes 2} ;$ le groupe engendré par $Q$ est isomorphe à $\mu_{3} ; C_{2}$ est la somme directe de ces deux groupes.

Le groupe $\mu_{9}^{\otimes 2}$ s'insère dans une suite exacte canonique de $G$-modules:

$$
0 \rightarrow Z / 3 Z \rightarrow \mu_{9}^{\otimes 2} \rightarrow Z / 3 Z \rightarrow 0
$$

(le sous-groupe de $\mu_{9}^{\otimes 2}$ formé des points annulés par 3 est isomorphe à $Z / 3 Z)$.

4.1.3. Soient $E_{2}^{\prime}, E_{2}^{\prime \prime}, E_{2}^{\prime \prime \prime}$ resp. le quotient de $E_{2}$ par $Z / 3 Z, \mu_{9}^{\otimes 2}, \mu_{3}$ resp. On obtient un diagramme canonique formé d'isogénies de degré 3 définies 
$\operatorname{sur} Q$ :

$$
E_{2}^{\prime \prime \prime} \leftarrow E_{2} \rightarrow E_{2}^{\prime} \rightarrow E_{2}^{\prime \prime}
$$

Proposition 4.1.3.1. a) Les courbes $E_{2}, E_{2}^{\prime}, E_{2}^{\prime \prime \prime}$ contiennent un point d'ordre 3 rationnel sur $\boldsymbol{Q}$.

b) L'anneau des endomorphismes de $E_{2}, E_{2}^{\prime}$, resp. $E_{2}^{\prime \prime}, E_{2}^{\prime \prime \prime}$ est isomorphe à l'anneau $O_{K}$ des entiers $d u$ corps $K$ des racines cubiques de l'unité, resp. $\grave{a}$ un ordre d'indice 3 dans $O_{K}$; en particulier:

$$
j\left(E_{2}\right)=j\left(E_{2}^{\prime}\right)=0 ; \quad j\left(E_{2}^{\prime \prime}\right)=j\left(E_{2}^{\prime \prime \prime}\right)=-3 \cdot 2^{15} \cdot 5^{3}
$$

j désignant la fonction invariant modulaire.

c) Les quatre courbes $E_{2}, E_{2}^{\prime}, E_{2}^{\prime \prime}, E_{2}^{\prime \prime \prime}$ appartiennent à quatre classes de $\mathbf{Q}$-isomorphie distinctes. La courbe $E_{2}^{\prime}$ est isomorphe sur $\boldsymbol{Q}$ à la courbe $E_{1}$.

Démonstration. a) Le groupe des points de $E_{2}$ annulé par 3 est isomorphe à $\boldsymbol{Z} / 3 \boldsymbol{Z} \oplus \boldsymbol{\mu}_{3} \subseteq \boldsymbol{\mu}_{9}^{\otimes 2} \oplus \boldsymbol{\mu}_{3}$.

Le quotient $E_{2}^{\prime}$ de $E_{2}$ par le sous-groupe isomorphe à $Z / 3 Z$ contient $\mu_{9}^{\otimes 2} /(Z / 3 Z) \cong Z / 3 Z$.

Enfin, le quotient $E_{2}^{\prime \prime \prime}$ de $E_{2}$ par $\mu_{3}$ contient $Z / 3 Z \subseteq \mu_{9}^{\otimes 2}$.

b) La courbe $E_{2}$ est associée au réseau $L_{2}$, où

$$
L_{2}=\omega_{2}^{-} \cdot\left[1, \tau_{2}\right] \text {. }
$$

D'après (3.3.1), et $\omega_{2}^{+}=-3 \omega_{1}^{+}, \omega_{2}^{-}=\omega_{1}^{-}$

$$
\tau_{2}=-\omega,
$$

ce qui montre que $\left[1, \tau_{2}\right]$ est isomorphe à $O_{K}$.

La courbe $E_{2}^{\prime}$ est associée au réseau:

$$
\begin{aligned}
L_{2}^{\prime} & =\omega_{2}^{-} \cdot\left[1, \frac{1-\omega}{3}\right] \\
& =\frac{1-\omega}{3} \cdot \omega_{2}^{-}[1, \omega] ;
\end{aligned}
$$

par conséquent, $L_{2}^{\prime}$ est homothétique au réseau $O_{K}$.

La courbe $E_{2}^{\prime \prime}$ est associée au réseau:

$$
L_{2}^{\prime \prime}=\omega_{2}^{-} \cdot\left[1, \frac{4-\omega}{9}\right]
$$




$$
=\omega_{2}^{-} \cdot\left(\frac{2 \omega+1}{9}\right) \cdot[1,3 \omega] .
$$

Enfin, la courbe $E_{2}^{\prime \prime \prime}$ est associée au réseau:

$$
L_{2}^{\prime \prime \prime}=\omega_{2}^{-} \cdot\left[\frac{1}{3}, \omega\right]=\frac{\omega_{2}^{-}}{3}[1,3 \omega] .
$$

On a donc: $j\left(E_{2}\right)=j\left(E_{2}^{\prime}\right)=0$;

$$
j\left(E_{2}^{\prime \prime}\right)=j\left(E_{2}^{\prime \prime \prime}\right)=-3 \cdot 2^{15} \cdot 5^{3} .
$$

c) La courbe $E_{2}$ n'est pas isomorphe sur $\boldsymbol{Q}$ à la courbe $E_{2}^{\prime}$. En effet, le quotient de $E_{2}$ par le sous-groupe $Z / 3 Z$ qu'elle contient est $E_{2}^{\prime}$, d'invariant nul, alors que le quotient de $E_{2}^{\prime}$ par son sous-groupe isomorphe à $Z / 3 Z$ est $E_{2}^{\prime \prime}$, dont l'invariant est non nul.

On sait que le graphe des 3-isogénies entre les courbes de conducteur 27 ne contient pas de cycle; donc $E_{2}^{\prime \prime}$ et $E_{2}^{\prime \prime \prime}$ ne sont pas $Q$-isomorphes.

La courbe $E_{1}$ est associée au réseau:

$$
L_{1}=\omega_{1}^{-} \cdot\left[1, \tau_{1}\right]
$$

d'après (3.3.1), on a:

$$
\tau_{1}=\frac{1}{2}\left(1+i \frac{\sqrt{3}}{3}\right)
$$

d'où

$$
L_{1}=\frac{\omega_{1}^{-}}{3}(2+\omega) \cdot[1, \omega] .
$$

La courbe $E_{1}$ coïncide donc avec la courbe $E_{2}$ ou la courbe $E_{2}^{\prime}$ (on pourrait aussi remarquer que le groupe des points de $E_{1}$ annulé par 3 est isomorphe à $Z / 3 Z \oplus \mu_{3}$, pour obtenir la même conclusion).

Le quotient de $E_{1}$ par le sous-groupe isomorphe à $\mu_{3}$ est associé au réseau:

$$
\frac{\omega_{1}^{-}}{3}\left[1,3 \tau_{1}\right]=\frac{\omega_{1}^{-}}{3}[1, \omega] .
$$

Ce quotient a donc un invariant nul. Ceci montre que $E_{1}$ est isomorphe à $E_{2}^{\prime}$, et non à $E_{2}$.

4.2. La variété abélienne $A$

Considérons la variété abélienne $A$ associée aux formes $f_{3}, f_{4}$, cf. 3.1.1.1. 
Cette variété est définie sur $\boldsymbol{Q}$, de dimension 2. On a:

$$
A(C) \cong C^{2} / L_{A}
$$

L'opérateur de Hecke $T(2)$ définit un endomorphisme de $A$, défini $\operatorname{sur} \boldsymbol{Q}$.

L'opérateur $R_{3}^{*}$ (cf. 2.2) définit un endomorphisme $R_{3}$ de $A$, défini sur le corps $K=\boldsymbol{Q}(\omega)$. Soit $\varepsilon$ l'automorphisme non trivial de $K$.

On a:

$$
\begin{aligned}
& R_{3}^{\varsigma}=-R_{3} ; \\
& R_{3} \circ T(2)+T(2) \circ R_{3}=0 \\
& R_{3}^{2}=-3 ; \quad T(2)^{2}=3 .
\end{aligned}
$$

Soit $\lambda=T(2)-R_{3}, \lambda^{s}=T(2)+R_{3}$.

Soit $B=\operatorname{Im} \lambda, B^{\varepsilon}=\operatorname{Im} \lambda^{\varepsilon}$. Alors $B$ et $B^{\varepsilon}$ sont des courbes elliptiques définies sur $K$.

Notons $K^{\prime}=Q\left(e^{2 \pi i / 9}\right)$.

Nous allons montrer:

Proposition 4.2.1. a) On $a B=\operatorname{Ker} \lambda, B^{s}=\operatorname{Ker} \lambda^{s}$.

b) Les points de 3-division de la courbe elliptique $B$ (resp. $B^{s}$ ) sont rationnels sur $K^{\prime}$. Il existe une base des points de 3-division de $B$ telle que l'action de $\mathrm{Gal}\left(K^{\prime} \mid K\right)$ soit donnée par la matrice

$$
\left(\begin{array}{ll}
1 & 1 \\
0 & 1
\end{array}\right)
$$

de $G L_{2}\left(F_{3}\right)$.

c) Soit $C$ le sous-groupe d'ordre 3 de $B$ formé de points rationnels sur $K$. Alors $B \cap B^{s}$ est le produit de $C$ par le groupe des points de 2division de $B$ (resp. $\left.B^{\varepsilon}\right)$.

d) On $a$ :

$$
\begin{aligned}
& B / C \cong B^{\varepsilon}, \\
& B^{\varepsilon} / C \cong B ;
\end{aligned}
$$

e) Soit i: $B \cap B^{s} \rightarrow B \times B^{s}$ l'application $b \mapsto(b,-b)$

et

$$
\begin{gathered}
j: B \times B^{s} \rightarrow A \\
\left(b, b^{\prime}\right) \mapsto b+b^{\prime} .
\end{gathered}
$$


La suite exacte:

$$
0 \longrightarrow B \cap B^{s} \stackrel{i}{\longrightarrow} B \times B^{s} \stackrel{j}{\longrightarrow} A \longrightarrow 0
$$

fait de $A$ la somme amalgamée de $B$ et $B^{*}$ le long de $B \cap B^{*}$. Le morphisme:

$$
B \times B^{e} \stackrel{\mathrm{pr}_{2}}{\longrightarrow} B^{e} \longrightarrow B^{e} / B \cap B^{e},
$$

resp.

$$
B \times B^{\mathrm{s}} \stackrel{\mathrm{pr}_{1}}{\longrightarrow} B \longrightarrow B / B \cap B^{\mathrm{s}},
$$

se factorise à travers $j: B \times B^{\mathfrak{s}} \rightarrow A$.

Identifiant $B^{\mathrm{s}} / B \cap B^{s} \grave{a} B$, resp. $B / B \cap B^{s} \grave{a} B^{s}$, le morphisme obtenu:

$$
A \rightarrow B
$$

resp.

$$
A \rightarrow B^{8}
$$

s'identifie à $\lambda$, resp. $\lambda^{s}$.

Remarque. La partie de (b) concernant le corps de rationalité des points de 3-division de $B$ est démontrée par Koike dans [3], en utilisant les traces des opérateurs de Hecke. Dans ce qui suit, nous démontrons la Prop. 4.2.1.

4.2.2. D'après 1.1, l'opérateur de Hecke $T(2)$ est induit par l'application:

$$
\left(z_{3}, z_{4}\right) \mapsto\left(\sqrt{3} \cdot z_{3},-\sqrt{3} \cdot z_{4}\right)
$$

de $C^{2}$ dans lui-même.

De:

$$
\begin{aligned}
& f_{3} \mid R_{3}^{*}=i \sqrt{3} \cdot f_{4}, \\
& f_{4} \mid R_{3}^{*}=i \sqrt{3} \cdot f_{3},
\end{aligned}
$$

résulte que $R_{3}$ est induit par l'application:

$$
\left(z_{3}, z_{4}\right) \mapsto\left(i \sqrt{3} \cdot z_{4}, i \sqrt{3} \cdot z_{3}\right)
$$

de $C^{2}$ dans lui-même.

On en déduit que $\lambda$ est induit par l'application $\tilde{\lambda}$ :

$$
\begin{gathered}
\tilde{\lambda}: C^{2} \rightarrow C^{2} \\
\left(z_{3}, z_{4}\right) \mapsto\left(\sqrt{3}\left(z_{3}-i z_{4}\right),-\sqrt{3}\left(z_{4}+i z_{3}\right)\right) .
\end{gathered}
$$


Il en résulte en particulier que l'image de $\tilde{\lambda}$ est isomorphe à $C$, et formée des couples $\left(z_{3},-i z_{3}\right)$, où $z_{3} \in C$.

Utilisons maintenant les notations de (3.3.3). Un calcul facile, utilisant les identités (3.3.2) montre que $\tilde{\lambda}$ correspond à l'application:

$$
M(a, b, c, d) \mapsto M(3(b+c), 3 d-a, a-3 d, b+c) .
$$

Rappelons que le réseau $L_{A}$ est défini par les conditions:

$$
\begin{aligned}
& a, b, c, d \in Z ; \\
& a \equiv b(\bmod 2) ; \\
& c \equiv d(\bmod 2) .
\end{aligned}
$$

On vérifie que

$$
\tilde{\lambda}\left(L_{A}\right) \subseteq L_{A}
$$

On voit aussi que l'image de $\tilde{\lambda}$ est caractérisée par les équations:

$$
\left\{\begin{array}{l}
a, b, c, d \in R ; \\
a=3 d ; \quad b+c=0 .
\end{array}\right.
$$

Posons

$$
2 \tau_{B}-1=\sqrt{3} \cdot \frac{\sqrt{3} \cdot \omega_{3}^{+}+\omega_{3}^{-}}{\sqrt{3} \cdot \omega_{3}^{+}-\omega_{3}^{-}}
$$

On a alors:

$$
M_{3}(3 d, b,-b, d)=\left(\sqrt{3} \cdot \omega_{3}^{+}-\omega_{3}^{-}\right) \cdot\left(-\frac{b+d}{2}+d \cdot \tau_{B}\right)
$$

Enfin, pour que $M(3 d, b,-b, d)$ soit un vecteur de $L_{A}$, il faut et il suffit que le vecteur $-(b+d) / 2+d \cdot \tau_{B}$ appartienne au réseau $\left[1, \tau_{B}\right]$. Ceci montre que la courbe $B$ est associée au réseau $\left[1, \tau_{B}\right]$.

Nous aurons besoin plus loin de l'expression de $\tilde{\lambda}^{\circ}$. Procédant comme pour $\tilde{\lambda}$, on montre que $\tilde{\lambda}^{\varepsilon}$ correspond à l'application:

$$
M(a, b, c, d) \mapsto M(3(c-b), a+3 d, a+3 d, b-c) .
$$

Posons:

$$
2 \tau_{B^{\varepsilon}}-1=\sqrt{3} \cdot \frac{\sqrt{3} \cdot \omega_{3}^{+}-\omega_{3}^{-}}{\sqrt{3} \cdot \omega_{3}^{+}+\omega_{3}^{-}}
$$

On a alors: 


$$
M_{3}(-3 d, b, b, d)=\left(\sqrt{3} \cdot \omega_{3}^{+}+\omega_{3}^{-}\right) \cdot\left(\frac{b+d}{2}-d \cdot \tau_{B^{s}}\right) .
$$

Pour que $M(-3 d, b, b, d)$ soit un vecteur de $L_{A}$, il faut et il suffit que le vecteur $(b+d) / 2-d \cdot \tau_{B^{s}}$ appartienne au réseau $\left[1, \tau_{B^{6}}\right]$; la courbe $B^{s}$ est donc associée à ce réseau.

Remarquons que l'on a l'identité:

$$
\left(2 \tau_{B}-1\right)\left(2 \tau_{B^{s}}-1\right)=3 .
$$

Il en résulte:

$$
\left\{\begin{array}{l}
{\left[1, \tau_{B^{6}}\right]=\left(2 \tau_{B^{6}}-1\right) \cdot\left[1, \frac{1+\tau_{B}}{3}\right] ;} \\
{\left[1, \tau_{B}\right]=\left(2 \tau_{B}-1\right) \cdot\left[1, \frac{1+\tau_{B^{6}}}{3}\right] .}
\end{array}\right.
$$

4.2.3. On sait que le noyau de $\lambda$ contient $B$, puisque $\lambda^{2}=0$. Montrons que $\operatorname{Ker} \lambda=B$.

On a:

$$
M^{-1}\left(\tilde{\lambda}^{-1}\left(L_{A}\right)\right)=\left\{\begin{array}{l|l}
(a, b, c, d) \in R^{4} & \begin{array}{l}
a-3 d \in Z \\
b+c \in Z \\
a-3 d \equiv b+c \quad(\bmod 2)
\end{array}
\end{array}\right\} .
$$

Pour tout élément $M(a, b, c, d)$ de $\tilde{\lambda}^{-1}\left(L_{A}\right)$, on a:

$$
M(a-3 d, b+c, 0,0) \in L_{A} .
$$

Par conséquent, tout élément de $\tilde{\lambda}^{-1}\left(L_{A}\right)$ est congru, modulo $L_{A}$, à un élément de la forme:

$$
M(3 d,-c, c, d)
$$

c'est-à-dire, d'après (4.2.2.0), à un élément de l'image de $\lambda_{\text {. }}$ En d'autres termes:

$$
\tilde{\lambda}\left(C^{2}\right)+L_{A}=\tilde{\lambda}^{-1}\left(L_{A}\right) .
$$

On a donc:

$$
\operatorname{Ker} \lambda=\tilde{\lambda}^{-1}\left(L_{A}\right) / L_{A} \cong \tilde{\lambda}\left(C^{2}\right) / \tilde{\lambda}\left(C^{2}\right) \cap L_{A}=B, \quad d \text { 'où (a). }
$$

4.2.4. La table 7 montre que les projections des pointes $0, \frac{1}{3}, \frac{2}{3}, \frac{1}{9}, \frac{4}{9}$, $\frac{7}{9}$ de $X_{0}\left(3^{4}\right)$ sur $A$ sont dans le noyau de $\lambda$, donc dans $B$. Ramenées dans le parallélogramme construit sur $1, \tau_{B}$, ces projections ont les coordonnées 
indiquées dans le tableau suivant:

\begin{tabular}{|l|c|c|c|c|c|c|}
\hline pointe & 0 & $\frac{1}{3}$ & $\frac{2}{3}$ & $\frac{1}{9}$ & $\frac{4}{9}$ & $\frac{7}{9}$ \\
\hline coordonnées & $(0,0)$ & $\left(\frac{1}{3}, \frac{1}{3}\right)$ & $\left(\frac{2}{3}, \frac{2}{3}\right)$ & $\left(\frac{2}{3}, \frac{1}{3}\right)$ & $\left(\frac{1}{3}, 0\right)$ & $\left(0, \frac{2}{3}\right)$ \\
\hline
\end{tabular}

On voit que les images de ces pointes engendrent le groupe des points de $B$ annulés par 3. Plus précisément, soient $P, Q$ resp. les images de $\frac{1}{3}, \frac{1}{9}$ resp. Soit $K^{\prime}=\boldsymbol{Q}\left(e^{2 \pi i / 9}\right)$; rappelons que $K=\boldsymbol{Q}(\omega)$, avec $\omega=e^{2 \pi i / 3}$. L'action du générateur [4] de $\mathrm{Gal}\left(K^{\prime} / K\right)$ sur les pointes est la suivante, cf. 4.1.1:

[4] laisse fixes $0, \frac{1}{3}, \frac{2}{3}$, et permute cycliquement les pointes $\frac{1}{9}, \frac{7}{9}, \frac{4}{9}$.

On a donc:

$$
\begin{gathered}
P^{[4]}=P ; \\
Q^{[4]}=P+Q,
\end{gathered}
$$

et, par rapport à la base $\{P, Q\}$, [4] est représenté par la matrice $\left(\begin{array}{ll}1 & 1 \\ 0 & 1\end{array}\right)$.

On a ainsi démontré (b). Ce résultat permet à Koike de déterminer une équation de la courbe $B$ (à $\varepsilon$-automorphisme près); cf. [3].

4.2.5. Soit $C$ le sous-groupe d'ordre 3 engendré par l'image de la pointe $\frac{1}{3}$. Les autres points annulés par 3 ne sont pas rationnels sur $K$.

Déterminons $B \cap B^{\varepsilon}$.

On doit déterminer:

$$
\left.\tilde{\lambda}^{-1}\left(L_{A}\right) \cap\left(\tilde{\lambda}^{s}\right)^{-1}\left(L_{A}\right) \quad \text { (modulo } L_{A}\right) .
$$

Pour que $M(a, b, c, d)$ soit dans cette intersection, il faut et il suffit que l'on ait:

$$
\text { (A) }\left\{\begin{array}{l}
a, b, c, d \in R^{4} ; \\
a-3 d \in Z ; \\
b+c \in Z ; \\
a+3 d \in Z ; \\
b-c \in Z ; \\
a-3 d \equiv b+c(\bmod 2) ; \\
a+3 d \equiv b-c(\bmod 2) .
\end{array}\right.
$$

Enfin, pour que $M(a, b, c, d)$ soit dans $L_{A}$, il faut et il suffit que: 


$$
\text { (B) }\left\{\begin{array}{l}
a, b, c, d \in Z ; \\
a \equiv b(\bmod 2) ; \\
c \equiv d(\bmod 2) .
\end{array}\right.
$$

Lemme 4.2.5.1. L'intersection $B \cap B^{*}$ est formée des classes $\bmod L_{A}$ des douze vecteurs $M(a, b, c, d)$, où $(a, b, c, d)$ est donné par le tableau suivant:

\begin{tabular}{|l|l|}
\hline$(0,0,0,0)$ & $(0,1,1,0)$ \\
$\left(\frac{1}{2}, \frac{1}{2}, \frac{3}{2}, \frac{1}{6}\right)$ & $\left(\frac{1}{2}, \frac{3}{2}, \frac{1}{2}, \frac{1}{6}\right)$ \\
$\left(0,0,1, \frac{1}{3}\right)$ & $\left(0,1,0, \frac{1}{3}\right)$ \\
$\left(\frac{1}{2}, \frac{1}{2}, \frac{1}{2}, \frac{1}{2}\right)$ & $\left(\frac{1}{2}, \frac{3}{2}, \frac{3}{2}, \frac{1}{2}\right)$ \\
$\left(0,0,0, \frac{2}{3}\right)$ & $\left(0,1,1, \frac{2}{3}\right)$ \\
$\left(\frac{1}{2}, \frac{1}{2}, \frac{3}{2}, \frac{5}{6}\right)$ & $\left(\frac{1}{2}, \frac{3}{2}, \frac{1}{2}, \frac{5}{6}\right)$ \\
\hline
\end{tabular}

Démonstration. On déduit des conditions (A): $2 a, 2 b, 2 c, 6 d \in Z$. Posons:

$$
2 a=\alpha, \quad 2 b=\beta, 2 c=\gamma, \quad 6 d=\delta .
$$

Les conditions (A) s'écrivent:

$$
\begin{array}{ll}
\alpha \equiv \beta \equiv \gamma \equiv \delta & (\bmod 2), \\
\delta \equiv \alpha-\beta-\gamma & (\bmod 4) .
\end{array}
$$

Ces congruences admettent 16 solutions $(\bmod 4)$.

Pour que $M(a, b, c, d)$ soit un élément de $L_{A}$, il faut et il suffit que:

$$
\begin{array}{lrr}
\alpha \equiv \beta \equiv \gamma \equiv 0(\bmod 2) ; & \delta \equiv 0(\bmod 6) ; \\
\alpha+\beta \equiv 0 & (\bmod 4) ; & 3 \gamma+\delta \equiv 0(\bmod 12) .
\end{array}
$$

Ces congruences admettent 108 solutions $(\bmod 12)$.

Par conséquent, $B \cap B^{s}$ contient $(16 \cdot 81) / 108=12$ éléments.

Il est facile de déterminer des représentants, en utilisant le fait que, quitte à modifier $M(a, b, c, d)$ par un vecteur de $L_{A}$, on peut supposer:

$$
0 \leq \alpha \leq 1 ; 0 \leq \beta, \gamma \leq 3 ; 0 \leq \delta \leq 5 \text {. }
$$

Rappelons que la courbe $B$ est associée au réseau $\left[1, \tau_{B}\right]$, cf. (4.2.2). Dans le parallélogramme construit sur $1, \tau_{B}$, les douze points de $B \cap B^{\text {e }}$ ont pour coordonnées respectives: 


\begin{tabular}{|l|l|}
\hline$(0,0)$ & $\left(\frac{1}{2}, 0\right)$ \\
$\left(\frac{2}{3}, \frac{1}{6}\right)$ & $\left(\frac{1}{6}, \frac{1}{6}\right)$ \\
$\left(\frac{5}{6}, \frac{1}{3}\right)$ & $\left(\frac{1}{3}, \frac{1}{3}\right)$ \\
$\left(\frac{1}{2}, \frac{1}{2}\right)$ & $\left(0, \frac{1}{2}\right)$ \\
$\left(\frac{2}{3}, \frac{2}{3}\right)$ & $\left(\frac{1}{6}, \frac{2}{3}\right)$ \\
$\left(\frac{1}{3}, \frac{5}{6}\right)$ & $\left(\frac{5}{6}, \frac{5}{6}\right)$ \\
\hline
\end{tabular}

On constate ainsi que $B \cap B^{*}$ contient les points d'ordre 2 de $B$, ainsi que les images des pointes $\frac{1}{3}$ et $\frac{2}{3}$; ces dernières engendrent le groupe $C$, qui est cyclique d'ordre 3 , cf. (4.2.4).

Par conséquent, $B \cap B^{*}$ est le produit de $C$ par le groupe des points de 2-torsion de $B$, resp. de $C$ par le groupe des points de 2-torsion de $B^{c}$. On peut schématiser comme suit la position des images des pointes de $X_{0}\left(3^{4}\right)$ dans $A$ :

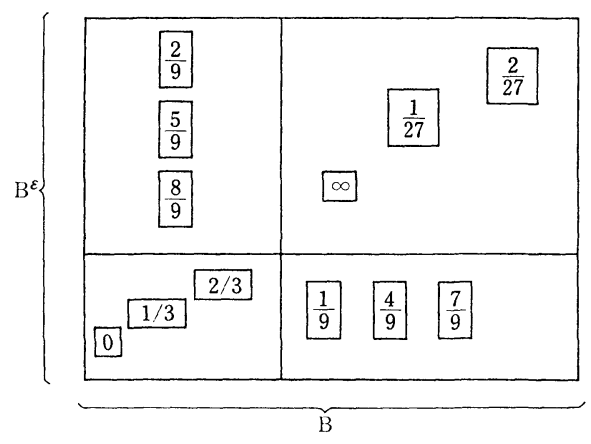

4.2.6. Considérons le quotient $B / C$. La courbe obtenue possède un point d'ordre 3 rationnel sur $K$, à savoir l'image de la pointe $\frac{1}{9}$, cf. (4.2.4). Elle vérifie donc les conditions du lemme du $\S 3$ de [3]. D'autre part, sa série $L$ coïncide avec celle de $B$. Les raisonnements de [3] montrent que cette courbe coïncide avec $B$ ou $B^{e}$. Ce ne peut pas être $B$, car $\operatorname{End}(B)$ $=Z$. C'est donc $B^{\circ}$.

Une vérification de ce résultat consiste à remarquer que le réseau définissant $B / C$ est, d'après 4.2 .4 , le réseau $\left[1,\left(1+\tau_{B}\right) / 3\right]$. Or ce dernier est homothétique au réseau $\left[1, \tau_{B}\right]$, cf. (4.2.2.2).

On peut aussi vérifier que le quotient de la courbe:

$$
y^{2}-3 x y+\omega y=x^{3}, \quad \omega=e^{2 \pi i / 3},
$$


par le sous-groupe engendré par le point $(0,0)$ est la courbe

$$
y^{2}-3 x y+\omega^{2} y=x^{3} ;
$$

(on utilise les formules de [9]; on obtient ainsi l'equation:

$$
y^{\prime 2}-3 x^{\prime} y^{\prime}+\omega y^{\prime}=x^{\prime 3}+15 \omega x^{\prime}+(7+34 \omega)
$$

d'où, posant:

$$
x=-\frac{1}{3} x^{\prime}-1, \quad y=-\frac{1}{3 i \sqrt{3}} y^{\prime}-\frac{1}{3}(\omega+2) x^{\prime}+\frac{4 \omega-10}{9}
$$

l'équation

$$
\left.y^{2}-3 x y+\omega^{2} y=x^{3}\right) .
$$

On a ainsi montré l'assertion (d) de la Prop. 4.2.1.

4.2.7. Considérons le morphisme:

$$
j: B \times B^{s} \rightarrow A .
$$

Ce morphisme est surjectif. Son noyau est l'ensemble des couples $(b,-b), b \in B \cap B^{s}$, donc l'image de $i$.

Considérons le morphisme composé:

$$
B \times B^{\varepsilon} \stackrel{\mathrm{pr}_{2}}{\longrightarrow} B^{\varepsilon} \longrightarrow B^{\varepsilon} / B \cap B^{\varepsilon} ;
$$

il est clair que son noyau contient l'image de $i$, d'où, par passage au quotient, un morphisme:

$$
A \rightarrow B^{\varepsilon} / B \cap B^{\varepsilon}
$$

Ce dernier est visiblement surjectif. D'autre part, son noyau est l'image par $j$ de $B \times\left(B \cap B^{c}\right)$, qui n'est autre que $B$.

L'unicité (à isomorphisme près) du quotient $A / B$ entraîne, tenant compte de l'assertion (a), l'existence d'un isomorphisme:

$$
\iota: B \cong B^{\varepsilon} / B \cap B^{s},
$$

ce qui fournit une nouvelle preuve de (d); d'autre part, le morphisme $A$ $\rightarrow B^{\natural} / B \cap B^{s}$ s'identifie à $\lambda$, pour un choix convenable de $\iota$. 
Table 1

Expression de $(\tilde{c}: \tilde{1}), 1 \leq c<81,(c, 3)=1$, en fonction de $(\tilde{c}: \tilde{1}), c \in \mathscr{B}$, où $\mathscr{B}=\{2,4,7,13,25,31,34,58\}$.

On note simplement (c) l'élément ( $\tilde{c}: \tilde{1})$; cf. 3.1

\begin{tabular}{|l|l|}
\hline$(1)=0$ & $(43)=-(31)$ \\
$(2)$ & $(44)=0$ \\
$(4)$ & $(46)=0$ \\
$(5)=(4)$ & $(47)=-(31)$ \\
$(7)$ & $(49)=-(34)$ \\
$(8)=0$ & $(50)=-(34)$ \\
$(10)=0$ & $(52)=-(13)$ \\
$(11)=(7)$ & $(53)=0$ \\
$(13)$ & $(55)=0$ \\
$(14)=(13)$ & $(56)=-(13)$ \\
$(16)=-(4)$ & $(58)$ \\
$(17)=0$ & $(59)=(58)$ \\
$(19)=0$ & $(61)=-(2)+(4)-(7)+(13)-(25)+(31)-(34)+(58)$ \\
$(20)=-(4)$ & $(62)=0$ \\
$(22)=-(7)$ & $(64)=0$ \\
$(23)=-(7)$ & $(65)=-(2)+(4)-(7)+(13)-(25)+(31)-(34)+(58)$ \\
$(25)$ & $(67)=-(25)$ \\
$(26)=0$ & $(68)=-(25)$ \\
$(28)=0$ & $(70)=-(58)$ \\
$(29)=(25)$ & $(71)=0$ \\
$(31)$ & $(73)=0$ \\
$(32)=(31)$ & $(74)=-(58)$ \\
$(34)$ & $(76)=(2)-(4)+(7)-(13)+(25)-(31)+(34)-(58)$ \\
$(35)=0$ & $(77)=(2)-(4)+(7)-(13)+(25)-(31)+(34)-(58)$ \\
$(37)=0$ & $(79)=(2)$ \\
$(38)=(34)$ & $(80)=0$ \\
$(40)=-(2)$ & \\
$(41)=-(2)$ & \\
\hline
\end{tabular}


Table $1^{\prime}$

Expression de $(\tilde{c}: \widetilde{3}), 1 \leq c<27,(c, 3)=1$, en fonction de $(\tilde{1}: \widetilde{3}),(\widetilde{2}: \widetilde{3})$ et $(\tilde{c}: \tilde{1}), c \in \mathscr{B} ;$ cf. Table 1 et 3.1 .

$$
\begin{aligned}
& (\tilde{4}: \widetilde{3})=(\widetilde{1}: \widetilde{3})+(2)-(4)+(7)-(13)+(25)-(31)+(34)-(58) \\
& (\widetilde{5}: \widetilde{3})=(\widetilde{2}: \tilde{3})+(34) \\
& (\widetilde{7}: \widetilde{3})=(\widetilde{1}: \widetilde{3})+(2)-(4)+(7)-(13)+(25)-(31)+(34) \\
& (\widetilde{8}: \widetilde{3})=(\widetilde{2}: \widetilde{3})-(31)+(34) \\
& (\widetilde{10}: \widetilde{3})=(\widetilde{1}: \widetilde{3})+(2)-(4)+(7)-(13)-(31)+(34) \\
& (\widetilde{11}: \widetilde{3})=(\widetilde{2}: \widetilde{3})+(25)-(31)+(34) \\
& (\widetilde{13}: \widetilde{3})=(\widetilde{1}: \widetilde{3})+(2)-(4)-(13)-(31)+(34) \\
& (\widetilde{14}: \widetilde{3})=(\widetilde{2}: \widetilde{3})-(2)+(4)-(7)+(13)+(58) \\
& (\widetilde{16}: \widetilde{3})=(\widetilde{1}: \widetilde{3})+(2)-(13)-(31)+(34) \\
& (\widetilde{17}: \widetilde{3})=(\widetilde{2}: \widetilde{3})-(2)+(4)-(7)+(13) \\
& (\widetilde{19}: \widetilde{3})=(\tilde{1}: \widetilde{3})+(2)-(31)+(34) \\
& (\widetilde{20}: \widetilde{3})=(\widetilde{2}: \widetilde{3})-(2)+(4)-(7) \\
& (\widetilde{22}: \widetilde{3})=(\tilde{1}: \widetilde{3})+(2)-(31) \\
& (\widetilde{23}: \widetilde{3})=(\widetilde{2}: \widetilde{3})-(2)+(4) \\
& (\widetilde{25}: \widetilde{3})=(\widetilde{1}: \widetilde{3})+(2) \\
& (\widetilde{26}: \widetilde{3})=(\widetilde{2}: \widetilde{3})-(2)
\end{aligned}
$$

Table 2

Conjugaison complexe (cf. 3.1).

On note $(\tilde{c}: \tilde{1})=(c)$.

$$
\begin{aligned}
& (\overline{2})=(2) \\
& (\overline{4})=(2)-(4)+(7)-(13)+(25)-(31)+(34)-(58) \\
& (\overline{7})=-(58) \\
& (\overline{13})=-(25) \\
& (\overline{25})=-(13) \\
& (\overline{31})=-(34) \\
& (\overline{34})=-(31) \\
& (\overline{58})=-(7)
\end{aligned}
$$


Table 3

On désigne par $(c)$ le vecteur $\xi((\tilde{c}: \tilde{1}))$; cf. 3.1.

$\gamma_{1}^{+}=(2)+(7)-(13)+(25)-(31)+(34)-(58)$
$\gamma_{2}^{+}=(7)-(58)$
$\gamma_{3}^{+}=(13)-(25)$
$\gamma_{4}^{+}=(31)-(34)$
$\gamma_{1}^{-}=-(2)+2(4)-(7)+(13)-(25)+(31)-(34)+(58)$
$\gamma_{2}^{-}=(7)+(58)$
$\gamma_{3}^{-}=(13)+(25)$
$\gamma_{4}^{-}=(31)+(34)$
$(2)=\gamma_{1}^{+}-\gamma_{2}^{+}+\gamma_{3}^{+}+\gamma_{4}^{+}$
$(4)=\frac{1}{2} \gamma_{1}^{+}+\frac{1}{2} \gamma_{1}^{-}$
$(7)=\frac{1}{2} \gamma_{2}^{+}+\frac{1}{2} \gamma_{2}^{-}$
$(13)=\frac{1}{2} \gamma_{3}^{+}+\frac{1}{2} \gamma_{3}^{-}$
$(25)=-\frac{1}{2} \gamma_{3}^{+}+\frac{1}{2} \gamma_{3}^{-}$
$(31)=\frac{1}{2} \gamma_{4}^{+}+\frac{1}{2} \gamma_{4}^{-}$
$(34)=-\frac{1}{2} \gamma_{4}^{+}+\frac{1}{2} \gamma_{4}^{-}$
$(58)=-\frac{1}{2} \gamma_{2}^{+}+\frac{1}{2} \gamma_{2}^{-}$

Table 4

On note:

$$
\begin{array}{ll}
\omega_{1}^{+}=\gamma_{1,1}^{+} ; & \omega_{1}^{-}=\gamma_{1,1}^{-} ; \\
\omega_{2}^{+}=-3 \omega_{1}^{+} ; & \omega_{2}^{-}=\omega_{1}^{-} ; \\
\omega_{3}^{+}=\gamma_{2,3}^{+} ; & \omega_{3}^{-}=\gamma_{2,3}^{-} ; \\
\omega_{4}^{+}=\gamma_{2,4}^{+} ; & \omega_{4}^{-}=\gamma_{2,4}^{-} .
\end{array}
$$

cf. 3.1.4. 


\begin{tabular}{|llll|}
\hline$\gamma_{1,1}^{+}=\omega_{1}^{+}$ & $\gamma_{1,2}^{+}=0$ & $\gamma_{1,3}^{+}=2 \omega_{3}^{+}$ & $\gamma_{1,4}^{+}=2 \omega_{4}^{+}$ \\
$\gamma_{2,1}^{+}=-\omega_{1}^{+}$ & $\gamma_{2,2}^{+}=\omega_{2}^{+}$ & $\gamma_{2,3}^{+}=\omega_{3}^{+}$ & $\gamma_{2,4}^{+}=\omega_{4}^{+}$ \\
$\gamma_{3,1}^{+}=-2 \omega_{1}^{+}$ & $\gamma_{3,2}^{+}=\omega_{2}^{+}$ & $\gamma_{3,3}^{+}=(\sqrt{3}-1) \omega_{3}^{+}$ & $\gamma_{3,4}^{+}=-(1+\sqrt{3}) \omega_{4}^{+}$ \\
$\gamma_{4,1}^{+}=\omega_{1}^{+}$ & $\gamma_{4,2}^{+}=0$ & $\gamma_{4,3}^{+}=-\omega_{3}^{+}$ & $\gamma_{4,4}^{+}=-\omega_{4}^{+}$ \\
\hline$\gamma_{1,1}^{-}=\omega_{1}^{-}$ & $\gamma_{1,2}^{-}=2 \omega_{2}^{-}$ & $\gamma_{1,3}^{-}=0$ & $\gamma_{1,4}^{-}=0$ \\
$\gamma_{2,1}^{-}=\omega_{1}^{-}$ & $\gamma_{2,2}^{-}=\omega_{2}^{-}$ & $\gamma_{2,3}^{-}=\omega_{3}^{-}$ & $\gamma_{2,4}^{-}=\omega_{4}^{-}$ \\
$\gamma_{3,1}^{-}=0$ & $\gamma_{3,2}^{-}=-\omega_{2}^{-}$ & $\gamma_{3,3}^{-}=(\sqrt{3}+1) \omega_{3}^{-}$ & $\gamma_{3,4}^{-}=(1-\sqrt{3}) \omega_{4}^{-}$ \\
$\gamma_{4,1}^{-}=\omega_{1}^{-}$ & $\gamma_{4,2}^{-}=0$ & $\gamma_{4,3}^{-}=-\omega_{3}^{-}$ & $\gamma_{4,4}^{-}=-\omega_{4}^{-}$ \\
\hline
\end{tabular}

Table 5

On pose:

$$
\{0, \pm z\}=\frac{1}{2}\left[X(z, k) \cdot \omega_{k}^{+} \pm Y(z, k) \cdot \omega_{k}^{-}\right], \quad 1 \leq k \leq 3 ;
$$

cf. 3.2 .

\begin{tabular}{|c|c|c|c|c|c|c|}
\hline pointe & $\infty$ & $\frac{1}{3}$ & $\frac{1}{9}$ & $\frac{2}{9}$ & $\frac{4}{9}$ & $\frac{1}{27}$ \\
\hline$X(z, 1)$ & $\frac{2}{3}$ & 1 & $-\frac{1}{3}$ & $\frac{2}{3}$ & $\frac{5}{3}$ & $\frac{2}{3}$ \\
\hline$Y(z, 1)$ & 0 & $\frac{1}{3}$ & $\frac{1}{3}$ & $\frac{2}{3}$ & $\frac{1}{3}$ & 0 \\
\hline$X(z, 2)$ & 0 & $-\frac{1}{3}$ & $\frac{4}{9}$ & $\frac{1}{9}$ & $-\frac{2}{9}$ & $-\frac{1}{3}$ \\
\hline$Y(z, 2)$ & 0 & $\frac{1}{3}$ & 0 & 1 & 0 & $-\frac{1}{3}$ \\
\hline$X(z, 3)$ & $\frac{2 \sqrt{3}}{3}$ & $\sqrt{3}$ & $\frac{\sqrt{3}}{3}$ & $\frac{1+\sqrt{3}}{\sqrt{3}}$ & $\frac{4-\sqrt{3}}{\sqrt{3}}$ & $-\frac{\sqrt{3}}{3}$ \\
\hline$Y(z, 3)$ & 0 & $\frac{\sqrt{3}}{3}$ & $\frac{2+\sqrt{3}}{3}$ & $\frac{1+\sqrt{3}}{3}$ & $-\frac{1}{3}$ & $\frac{\sqrt{3}}{3}$ \\
\hline
\end{tabular}

Table 6

Soit $z \in C^{4} ; z$ s'écrit de façon unique:

$$
z=\frac{1}{2} \sum_{k=1}^{4}\left(x_{k} \gamma_{k}^{+}+y_{k} \gamma_{k}^{-}\right)
$$

où $x_{k}, y_{k} \in R, 1 \leq k \leq 4$. 
On note:

$$
\pi_{i}(z)=\frac{1}{2}\left(a_{i} \omega_{i}^{+}+b_{i} \omega_{i}^{-}\right), \quad i=1,2,
$$

et

$$
\pi_{A}(z)=\left(M_{3}(a, b, c, d), M_{4}(a, b,-c,-d)\right)
$$

(cf. 3.3).

$$
\begin{array}{l|l}
a_{1}=x_{1}-x_{2}-2 x_{3}+x_{4} ; & 3 x_{1}=a_{1}+a+3 c ; \\
b_{1}=y_{1}+y_{2}+y_{4} ; & 3 x_{2}=3 a_{2}-3 c ; \\
a_{2}=x_{2}+x_{3} ; & 3 x_{3}=3 c ; \\
b_{2}=2 y_{1}+y_{2}-y_{3} ; & 3 x_{4}=2 a_{1}+3 a_{2}-a \\
a=2 x_{1}+x_{2}-x_{3}-x_{4} ; & 3 y_{1}=-b_{1}+2 b_{2}-b+3 d ; \\
b=y_{2}+y_{3}-y_{4} ; & 3 y_{2}=2 b_{1}-b_{2}+2 b-3 d ; \\
c=x_{3} ; & 3 y_{3}=3 d ; \\
d=y_{3} . & 3 y_{4}=2 b_{1}-b_{2}-b .
\end{array}
$$

Table 7

Soit $z \in \mathfrak{S}^{*}$ une pointe de $X_{0}\left(3^{4}\right)$.

On note:

$$
\begin{gathered}
\{0, z\}_{f_{j}}=\frac{1}{2}\left(a_{j} \omega_{j}^{+}+b_{j} \omega_{j}^{-}\right), \quad j=1,2 . \\
\left(\{0, z\}_{f_{3}},\{0, z\}_{f_{4}}\right)=M(a, b, c, d),
\end{gathered}
$$

cf. (3.3.3).

\begin{tabular}{|c|c|c|c|c|c|c|c|c|}
\hline pointe & $a_{1}$ & $b_{1}$ & $a_{2}$ & $b_{2}$ & $a$ & $b$ & $c$ & $d$ \\
\hline$\infty$ & $\frac{2}{3}$ & 0 & 0 & 0 & 0 & 0 & $\frac{2}{3}$ & 0 \\
\hline$\frac{1}{3}$ & 1 & $\frac{1}{3}$ & $-\frac{1}{3}$ & $\frac{1}{3}$ & 0 & 0 & 1 & $\frac{1}{3}$ \\
\hline$\frac{1}{9}$ & $-\frac{1}{3}$ & $\frac{1}{3}$ & $\frac{4}{9}$ & 0 & 0 & $\frac{2}{3}$ & $\frac{1}{3}$ & $\frac{1}{3}$ \\
\hline$\frac{2}{9}$ & $\frac{2}{3}$ & $\frac{2}{3}$ & $\frac{1}{9}$ & 1 & 1 & $\frac{1}{3}$ & $\frac{1}{3}$ & $\frac{1}{3}$ \\
\hline$\frac{4}{9}$ & $\frac{5}{3}$ & $\frac{1}{3}$ & $-\frac{2}{9}$ & 0 & -1 & $-\frac{1}{3}$ & $\frac{4}{3}$ & 0 \\
\hline$\frac{1}{27}$ & $\frac{2}{3}$ & 0 & $-\frac{1}{3}$ & $-\frac{1}{3}$ & 0 & 0 & $-\frac{1}{3}$ & $\frac{1}{3}$ \\
\hline
\end{tabular}




\section{BIBLIOGRAPHIE}

[1] T. Hadano, On the conductor of an elliptic curve with a rational point of order 2. Nagoya Math. J., 53 (1974), 199-210.

[2] T. Honda and I. Miyawaki, Zeta-functions of elliptic curves of 2-power conductor. J. Math. Soc. Japan, 26, $\mathrm{n}^{\circ} 2$ (1974), 362-373.

[ 3 ] M. Koike, On certain Abelian varieties obtained from newforms of weight 2 on $\Gamma_{0}\left(3^{4}\right)$ and $\Gamma_{0}\left(3^{5}\right)$, Nagoya Math. J., 62 (1976), 29-39.

[4] G. Ligozat, Courbes modulaires de genre 1. Bull. Soc. Math. France, Suppl., Mém. $\mathrm{N}^{\circ} 43,80 \mathrm{p}(1975)$.

[5] - Courbes modulaires de niveau 11, in Modular Functions of one variable V. Lecture Notes in Math. 601, 149-237, Berlin-Heidelberg-New York, Springer 1977.

[6] G. Shimura, Introduction to the arithmetic theory of automorphic functions. Publ. Math. Soc. Japan, $\mathrm{N}^{\circ}$ 11, Iwanami Shoten and Princeton University Press (1971).

[ 7 ] — On elliptic curves with complex multiplication as factors of the jacobians of modular function fields. Nagoya Math. J., 43 (1971), 199-208.

[8] - On the factors of the jacobian variety of a modular function field. J. Math. Soc. Japan, 25, $\mathrm{N}^{\circ} 3(1973)$, 523-544.

[ 9 ] J. Vélu, Isogénies entre courbes elliptiques. C. R. Acad. Sci. Paris, 273 (1971), 238-241.

Université de Paris-Sud 